\title{
Improving on Adjusted R-Squared Supplement
}

Julian D. Karch

Leiden University

May 1, 2020

In the following, I provide bias and mean squared error (MSE) for all design cells. 
Table S3: Results for sample size $N=10$ and number of predictors $p=2$.

\begin{tabular}{|c|c|c|c|c|c|c|c|c|c|c|c|c|c|c|c|c|c|c|c|c|c|}
\hline$\rho^{2}$ & What & $R^{2}$ & $\mathrm{E}$ & OPE & $\mathrm{S}$ & W & OP1 & OP2 & OP5 & $\mathrm{P}$ & C & ML & $\mathrm{S}^{+}$ & $\mathrm{E}^{+}$ & $\mathrm{W}^{+}$ & $\mathrm{OP} 1^{+}$ & $\mathrm{OP}{ }^{+}$ & $\mathrm{OP}^{+}$ & $\mathrm{P}^{+}$ & $\mathrm{C}^{+}$ & $\mathrm{OPE}^{+}$ \\
\hline 0.00 & BIAS & 0.222 & -0.001 & $\begin{array}{l}-0.001 \\
0.078\end{array}$ & 0.027 & 0.124 & 0.080 & 0.036 & 0.006 & -0.002 & 0.141 & 0.115 & 0.101 & 0.092 & 0.147 & 0.134 & $\begin{array}{l}0.121 \\
0.48\end{array}$ & 0.116 & 0.107 & $\begin{array}{l}0.169 \\
0.068\end{array}$ & 0.115 \\
\hline 0.01 & BIAS & 0.218 & -0.003 & -0.001 & 0,025 & 0.122 & 0.078 & 0.036 & & רח 000 & 0.139 & 011 & 0007 & 0087 & 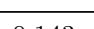 & & 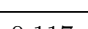 & & & & (1) \\
\hline 0.01 & MSE & 0.080 & 0.054 & 0.080 & 0.051 & $\begin{array}{l}0.122 \\
0.056\end{array}$ & $\begin{array}{l}0.078 \\
0.062\end{array}$ & $\begin{array}{l}0.056 \\
0.068\end{array}$ & $\begin{array}{l}0.006 \\
0.077\end{array}$ & $\begin{array}{l}-0.002 \\
0.072\end{array}$ & $\begin{array}{l}0.139 \\
0.072\end{array}$ & 0.044 & 0.038 & 0.034 & $\begin{array}{l}0.143 \\
0.054\end{array}$ & $\begin{array}{l}0.130 \\
0.053\end{array}$ & 0.049 & $\begin{array}{l}0.112 \\
0.048\end{array}$ & $\begin{array}{l}0.104 \\
0.044\end{array}$ & $\begin{array}{l}0.166 \\
0.069\end{array}$ & 0.112 \\
\hline 0.05 & BIAS & 0.204 & -0.009 & -0.001 & 0.017 & 0.111 & 0.072 & 0.032 & 0.006 & -0.004 & 0.132 & 0.096 & 0.080 & 0.069 & 0.129 & 0.117 & 0.103 & 0.098 & 0.088 & 0.155 & 0.098 \\
\hline 0.05 & MSE & 0.078 & 0.060 & 0.087 & 0.057 & 0.058 & 0.067 & 0.074 & 0.084 & 0.079 & 0.076 & 0.047 & 0.040 & 0.037 & 0.055 & 0.056 & 0.052 & 0.051 & 0.048 & 0.071 & 0.051 \\
\hline 0.10 & BIAS & 0.187 & -0.017 & 0.000 & 0.008 & 0.097 & 0.064 & 0.029 & 0.005 & -0.006 & 0.123 & 0.078 & 0.061 & 0.049 & 0.112 & 0.101 & 0.088 & 0.082 & 0.071 & 0.142 & 0.082 \\
\hline 0.10 & MSE & 0.075 & 0.068 & 0.094 & 0.064 & 0.061 & 0.072 & 0.081 & 0.091 & 0.086 & 0.079 & 0.050 & 0.044 & 0.042 & 0.057 & 0.059 & 0.057 & 0.056 & 0.053 & 0.073 & 0.056 \\
\hline 0.20 & BIAS & 0.154 & -0.031 & 0.000 & -0.008 & 0.073 & 0.050 & 0.022 & 0.004 & -0.009 & 0.106 & 0.047 & 0.027 & 0.013 & 0.082 & 0.074 & 0.061 & 0.055 & 0.043 & 0.118 & 0.055 \\
\hline 0.20 & MSE & 0.070 & 0.078 & 0.101 & 0.073 & 0.064 & 0.078 & 0.088 & 0.098 & 0.095 & 0.081 & 0.057 & 0.053 & 0.052 & 0.060 & 0.065 & 0.065 & 0.065 & 0.063 & 0.075 & 0.065 \\
\hline 0.30 & BIAS & 0.123 & -0.042 & 0.000 & -0.022 & 0.051 & 0.038 & 0.016 & 0.003 & -0.011 & 0.090 & 0.022 & 0.000 & -0.014 & 0.056 & 0.052 & 0.040 & 0.035 & 0.022 & 0.097 & 0.034 \\
\hline 0.30 & MSE & 0.064 & 0.083 & 0.100 & 0.077 & 0.064 & 0.079 & 0.089 & 0.097 & 0.097 & 0.079 & 0.061 & 0.060 & 0.061 & 0.061 & 0.068 & 0.070 & 0.071 & 0.070 & 0.074 & 0.071 \\
\hline 0.40 & BIAS & 0.094 & -0.050 & 0.000 & -0.032 & 0.031 & 0.027 & 0.010 & 0.002 & -0.012 & 0.075 & 0.002 & -0.020 & -0.034 & 0.034 & 0.035 & 0.024 & 0.020 & 0.007 & 0.079 & 0.020 \\
\hline 0.40 & MSE & 0.057 & 0.082 & 0.092 & 0.076 & 0.062 & 0.074 & 0.083 & 0.090 & 0.091 & 0.072 & 0.062 & 0.064 & 0.066 & 0.059 & 0.067 & 0.070 & 0.071 & 0.072 & 0.069 & 0.071 \\
\hline 0.50 & BIAS & 0.069 & -0.054 & 0.000 & -0.039 & 0.015 & 0.018 & 0.006 & 0.001 & -0.012 & 0.061 & -0.013 & -0.033 & -0.046 & 0.016 & 0.022 & 0.013 & 0.010 & -0.002 & 0.063 & 0.010 \\
\hline 0.50 & MSE & 0.049 & 0.075 & 0.078 & 0.070 & 0.056 & 0.065 & 0.072 & 0.077 & 0.079 & 0.062 & 0.059 & 0.063 & 0.066 & 0.054 & 0.061 & 0.064 & 0.066 & 0.067 & 0.060 & 0.066 \\
\hline 0.60 & BIAS & 0.046 & -0.055 & 0.000 & -0.042 & 0.002 & 0.010 & 0.003 & 0.000 & -0.010 & 0.048 & -0.023 & -0.039 & -0.051 & 0.003 & 0.012 & 0.006 & 0.004 & -0.006 & 0.049 & 0.004 \\
\hline 0.60 & MSE & 0.039 & 0.064 & 0.060 & 0.059 & 0.046 & 0.052 & 0.057 & 0.060 & 0.063 & 0.048 & 0.050 & 0.056 & 0.059 & 0.046 & 0.050 & 0.053 & 0.054 & 0.057 & 0.047 & 0.054 \\
\hline 0.70 & BIAS & 0.028 & -0.050 & 0.000 & -0.040 & -0.006 & 0.005 & 0.001 & 0.000 & -0.008 & 0.036 & -0.028 & -0.040 & -0.049 & -0.006 & 0.006 & 0.002 & 0.001 & -0.007 & 0.036 & 0.001 \\
\hline 0.70 & MSE & 0.028 & 0.047 & 0.041 & 0.044 & 0.034 & 0.037 & 0.039 & 0.041 & 0.043 & 0.033 & 0.038 & 0.043 & 0.046 & 0.034 & 0.036 & 0.038 & 0.039 & 0.041 & 0.033 & 0.039 \\
\hline 0.80 & BIAS & 0.013 & -0.040 & 0.000 & -0.034 & -0.010 & 0.002 & 0.000 & 0.000 & -0.005 & 0.024 & -0.027 & -0.034 & -0.040 & -0.010 & 0.002 & 0.000 & 0.000 & -0.005 & 0.024 & 0.000 \\
\hline 0.80 & MSE & 0.016 & 0.028 & 0.022 & 0.026 & 0.020 & 0.020 & 0.021 & 0.022 & 0.023 & 0.018 & 0.023 & 0.026 & 0.028 & 0.020 & 0.020 & 0.021 & 0.021 & 0.023 & 0.018 & 0.021 \\
\hline 0.90 & BIAS & 0.003 & -0.024 & 0.000 & -0.021 & -0.009 & 0.000 & 0.000 & 0.000 & -0.002 & 0.013 & -0.019 & -0.021 & -0.024 & -0.009 & 0.000 & 0.000 & 0.000 & -0.002 & 0.013 & 0.000 \\
\hline 0.90 & MSE & 0.005 & 0.010 & 0.007 & 0.009 & 0.007 & 0.006 & 0.006 & 0.007 & 0.007 & 0.005 & 0.008 & 0.009 & 0.010 & 0.007 & 0.006 & 0.006 & 0.006 & 0.007 & 0.005 & 0.006 \\
\hline
\end{tabular}


Table S4: Results for sample size $N=10$ and number of predictors $p=5$.

\begin{tabular}{|c|c|c|c|c|c|c|c|c|c|c|c|c|c|c|c|c|c|c|c|c|c|}
\hline$\rho^{2}$ & What & $R^{2}$ & $\mathrm{E}$ & OPE & $\mathrm{S}$ & $\mathrm{W}$ & OP1 & OP2 & OP5 & $\mathrm{P}$ & $\mathrm{C}$ & ML & $\mathrm{S}^{+}$ & $\mathrm{E}^{+}$ & $\mathrm{W}^{+}$ & $\mathrm{OP} 1^{+}$ & $\mathrm{OP}{ }^{+}$ & $\mathrm{OP}^{+}$ & $\mathrm{P}^{+}$ & $\mathrm{C}^{+}$ & $\mathrm{OPE}^{+}$ \\
\hline 0.00 & BIAS & 0.556 & 0.001 & 0.002 & 0.112 & 0.201 & 0.082 & 0.039 & 0.009 & -0.090 & 0.066 & 0.247 & 0.240 & 0.200 & 0.280 & 0.248 & 0.242 & 0.240 & 0.213 & 0.260 & 0.240 \\
\hline 0.00 & MSE & 0.354 & 0.227 & 0.349 & 0.192 & 0.186 & 0.243 & 0.286 & 0.331 & 0.402 & 0.294 & 0.137 & 0.132 & 0.108 & 0.156 & 0.141 & 0.139 & 0.138 & 0.123 & 0.153 & 0.138 \\
\hline 0.01 & BIAS & 0.550 & 0.000 & 0.002 & 0.110 & 0.198 & 0.081 & 0.038 & 0.009 & -0.089 & 0.065 & 0.241 & 0.234 & 0.194 & 0.275 & 0.243 & 0.236 & 0.234 & 0.207 & 0.255 & 0.234 \\
\hline 0.01 & MSE & 0.347 & 0.227 & 0.345 & 0.191 & 0.184 & 0.241 & 0.283 & 0.328 & 0.399 & 0.292 & 0.135 & 0.130 & 0.107 & 0.154 & 0.139 & 0.137 & 0.136 & 0.122 & 0.151 & 0.136 \\
\hline 0.05 & BIAS & 0.525 & -0.007 & 0.002 & 0.099 & 0.184 & 0.074 & 0.035 & 0.008 & -0.086 & 0.062 & 0.219 & 0.212 & 0.170 & 0.253 & 0.221 & 0.214 & 0.213 & 0.184 & 0.233 & 0.213 \\
\hline 0.05 & MSE & 0.319 & 0.223 & 0.332 & 0.186 & 0.177 & 0.235 & 0.275 & 0.316 & 0.387 & 0.282 & 0.127 & 0.123 & 0.102 & 0.144 & 0.132 & 0.130 & 0.130 & 0.117 & 0.144 & 0.130 \\
\hline 0.10 & BIAS & 0.493 & -0.015 & 0.002 & 0.087 & 0.168 & 0.067 & 0.031 & 0.007 & -0.082 & 0.058 & 0.193 & 0.185 & 0.142 & 0.228 & 0.195 & 0.188 & 0.187 & 0.157 & 0.208 & 0.186 \\
\hline 0.10 & MSE & 0.286 & 0.218 & 0.314 & 0.180 & 0.168 & 0.226 & 0.264 & 0.301 & 0.370 & 0.270 & 0.119 & 0.115 & 0.097 & 0.134 & 0.125 & 0.123 & 0.123 & 0.112 & 0.136 & 0.123 \\
\hline 0.20 & BIAS & 0.431 & -0.029 & 0.002 & 0.063 & 0.137 & 0.052 & 0.023 & 0.006 & -0.073 & 0.051 & 0.144 & 0.136 & 0.090 & 0.180 & 0.147 & 0.141 & 0.139 & 0.108 & 0.163 & 0.139 \\
\hline 0.20 & MSE & 0.226 & 0.205 & 0.277 & 0.165 & 0.149 & 0.205 & 0.237 & 0.267 & 0.331 & 0.242 & 0.107 & 0.104 & 0.092 & 0.117 & 0.114 & 0.113 & 0.113 & 0.107 & 0.124 & 0.113 \\
\hline 0.30 & BIAS & 0.371 & -0.041 & 0.002 & 0.042 & 0.108 & 0.039 & 0.017 & 0.004 & -0.064 & 0.045 & 0.101 & 0.093 & 0.045 & 0.137 & 0.106 & 0.100 & 0.098 & 0.067 & 0.123 & 0.098 \\
\hline 0.30 & MSE & 0.174 & 0.188 & 0.236 & 0.149 & 0.131 & 0.180 & 0.207 & 0.230 & 0.287 & 0.210 & 0.098 & 0.097 & 0.091 & 0.103 & 0.105 & 0.105 & 0.106 & 0.104 & 0.114 & 0.106 \\
\hline 0.40 & BIAS & 0.312 & -0.049 & 0.002 & 0.024 & 0.081 & 0.028 & 0.012 & 0.003 & -0.054 & 0.040 & 0.065 & 0.056 & 0.009 & 0.100 & 0.072 & 0.066 & 0.064 & 0.034 & 0.090 & 0.064 \\
\hline 0.40 & MSE & 0.129 & 0.165 & 0.193 & 0.129 & 0.111 & 0.153 & 0.173 & 0.189 & 0.237 & 0.174 & 0.090 & 0.089 & 0.091 & 0.090 & 0.096 & 0.097 & 0.098 & 0.101 & 0.103 & 0.098 \\
\hline 0.50 & BIAS & 0.254 & -0.053 & 0.001 & 0.009 & 0.058 & 0.019 & 0.008 & 0.002 & -0.043 & 0.035 & 0.036 & 0.028 & -0.018 & 0.068 & 0.044 & 0.040 & 0.038 & 0.010 & 0.064 & 0.038 \\
\hline 0.50 & MSE & 0.091 & 0.138 & 0.149 & 0.107 & 0.090 & 0.122 & 0.137 & 0.147 & 0.185 & 0.136 & 0.080 & 0.081 & 0.087 & 0.076 & 0.085 & 0.087 & 0.088 & 0.095 & 0.089 & 0.088 \\
\hline 0.60 & BIAS & 0.199 & -0.053 & 0.001 & -0.003 & 0.038 & 0.012 & 0.005 & 0.002 & -0.032 & 0.030 & 0.014 & 0.007 & -0.035 & 0.042 & 0.024 & 0.021 & 0.020 & -0.004 & 0.045 & 0.020 \\
\hline 0.60 & MSE & 0.060 & 0.107 & 0.106 & 0.083 & 0.068 & 0.091 & 0.099 & 0.105 & 0.132 & 0.097 & 0.066 & 0.068 & 0.078 & 0.061 & 0.070 & 0.072 & 0.073 & 0.081 & 0.072 & 0.073 \\
\hline 0.70 & BIAS & 0.145 & -0.049 & 0.001 & -0.010 & 0.021 & 0.006 & 0.003 & 0.001 & -0.021 & 0.025 & -0.001 & -0.006 & -0.042 & 0.023 & 0.011 & 0.009 & 0.008 & -0.010 & 0.030 & 0.008 \\
\hline 0.70 & MSE & 0.035 & 0.074 & 0.067 & 0.057 & 0.046 & 0.059 & 0.064 & 0.066 & 0.083 & 0.061 & 0.049 & 0.051 & 0.062 & 0.044 & 0.051 & 0.052 & 0.053 & 0.061 & 0.051 & 0.053 \\
\hline 0.80 & BIAS & 0.094 & -0.039 & 0.001 & -0.013 & 0.009 & 0.003 & 0.001 & 0.001 & -0.011 & 0.019 & -0.008 & -0.012 & -0.038 & 0.009 & 0.004 & 0.003 & 0.002 & -0.008 & 0.020 & 0.002 \\
\hline 0.80 & MSE & 0.017 & 0.041 & 0.033 & 0.032 & 0.026 & 0.031 & 0.032 & 0.033 & 0.040 & 0.030 & 0.029 & 0.030 & 0.038 & 0.025 & 0.029 & 0.030 & 0.030 & 0.035 & 0.027 & 0.030 \\
\hline 0.90 & BIAS & 0.045 & -0.023 & 0.000 & -0.010 & 0.001 & 0.001 & 0.000 & 0.000 & -0.003 & 0.012 & -0.008 & -0.010 & -0.023 & 0.001 & 0.001 & 0.001 & 0.001 & -0.003 & 0.012 & 0.001 \\
\hline 0.90 & MSE & 0.005 & 0.013 & 0.009 & 0.010 & 0.008 & 0.009 & 0.009 & 0.009 & 0.011 & 0.008 & 0.010 & 0.010 & 0.013 & 0.008 & 0.009 & 0.009 & 0.009 & 0.010 & 0.008 & 0.009 \\
\hline
\end{tabular}


Table S5: Results for sample size $N=20$ and number of predictors $p=2$.

\begin{tabular}{|c|c|c|c|c|c|c|c|c|c|c|c|c|c|c|c|c|c|c|c|c|c|}
\hline$\rho^{2}$ & What & $R^{2}$ & $\mathrm{E}$ & OPE & $\mathrm{S}$ & $\mathrm{W}$ & OP1 & OP2 & OP5 & $\mathrm{P}$ & C & ML & $\mathrm{S}^{+}$ & $\mathrm{E}^{+}$ & $\mathrm{w}^{+}$ & $\mathrm{OP} 1^{+}$ & $\mathrm{OP} 2^{+}$ & $\mathrm{OP}^{+}$ & $\mathrm{P}^{+}$ & $\mathrm{C}^{+}$ & $\mathrm{OPE}^{+}$ \\
\hline 0.00 & BIAS & 0.106 & 0.000 & 0.000 & 0.006 & 0.056 & 0.020 & 0.006 & 0.001 & 0.003 & 0.061 & 0.052 & 0.043 & 0.041 & 0.067 & 0.052 & 0.047 & 0.046 & 0.046 & 0.073 & 0.046 \\
\hline 0.00 & MSE & 0.020 & 0.011 & 0.014 & 0.011 & 0.013 & 0.013 & 0.013 & 0.014 & 0.013 & 0.015 & 0.011 & 0.008 & 0.008 & 0.013 & 0.010 & 0.010 & 0.009 & 0.009 & 0.015 & 0.009 \\
\hline 0.01 & BIAS & 0.104 & 0.000 & 0.001 & 0.005 & 0.055 & 0.020 & 0.006 & 0.001 & 0.002 & 0.061 & 0.049 & 0.040 & 0.037 & 0.065 & 0.049 & 0.044 & 0.043 & 0.043 & 0.071 & 0.043 \\
\hline 0.01 & MSE & 0.021 & 0.013 & 0.015 & 0.012 & 0.014 & 0.014 & 0.015 & 0.015 & 0.015 & 0.017 & 0.011 & 0.009 & 0.009 & 0.014 & 0.011 & 0.011 & 0.010 & 0.010 & 0.016 & 0.010 \\
\hline 0.05 & BIAS & 0.096 & -0.004 & 0.001 & 0.002 & 0.049 & 0.018 & 0.005 & 0.001 & 0.002 & 0.058 & 0.040 & 0.027 & 0.025 & 0.056 & 0.040 & 0.034 & 0.033 & 0.032 & 0.065 & 0.033 \\
\hline 0.05 & MSE & 0.023 & 0.018 & 0.021 & 0.017 & 0.018 & 0.019 & 0.020 & 0.021 & 0.020 & 0.021 & 0.015 & 0.013 & 0.012 & 0.017 & 0.016 & 0.015 & 0.015 & 0.015 & 0.020 & 0.015 \\
\hline 0.10 & BIAS & 0.087 & -0.008 & 0.001 & -0.003 & 0.042 & 0.016 & 0.004 & 0.001 & 0.001 & 0.054 & 0.030 & 0.015 & 0.012 & 0.047 & 0.031 & 0.024 & 0.023 & 0.022 & 0.059 & 0.023 \\
\hline 0.10 & MSE & 0.026 & 0.023 & 0.027 & 0.023 & 0.022 & 0.025 & 0.026 & 0.027 & 0.026 & 0.026 & 0.020 & 0.018 & 0.017 & 0.021 & 0.021 & 0.021 & 0.021 & 0.020 & 0.025 & 0.021 \\
\hline 0.20 & BIAS & 0.070 & -0.015 & 0.000 & -0.011 & 0.030 & 0.012 & 0.003 & 0.000 & 0.000 & 0.047 & 0.015 & -0.003 & -0.007 & 0.032 & 0.018 & 0.012 & 0.010 & 0.008 & 0.049 & 0.010 \\
\hline 0.20 & MSE & 0.029 & 0.031 & 0.034 & 0.030 & 0.028 & 0.032 & 0.034 & 0.034 & 0.034 & 0.032 & 0.027 & 0.027 & 0.027 & 0.027 & 0.029 & 0.030 & 0.030 & 0.029 & 0.031 & 0.030 \\
\hline 0.30 & BIAS & 0.055 & -0.021 & 0.000 & -0.017 & 0.019 & 0.008 & 0.002 & 0.000 & -0.001 & 0.040 & 0.005 & -0.014 & -0.018 & 0.020 & 0.011 & 0.005 & 0.004 & 0.002 & 0.040 & 0.004 \\
\hline 0.30 & MSE & 0.030 & 0.034 & 0.037 & 0.034 & 0.030 & 0.035 & 0.036 & 0.037 & 0.036 & 0.034 & 0.031 & 0.032 & 0.032 & 0.030 & 0.033 & 0.034 & 0.035 & 0.034 & 0.033 & 0.035 \\
\hline 0.40 & BIAS & 0.041 & -0.024 & 0.000 & -0.021 & 0.010 & 0.006 & 0.001 & 0.000 & -0.002 & 0.034 & -0.003 & -0.020 & -0.023 & 0.010 & 0.006 & 0.002 & 0.001 & -0.001 & 0.034 & 0.001 \\
\hline 0.40 & MSE & 0.028 & 0.034 & 0.035 & 0.033 & 0.030 & 0.034 & 0.035 & 0.035 & 0.035 & 0.032 & 0.030 & 0.033 & 0.033 & 0.030 & 0.033 & 0.034 & 0.034 & 0.034 & 0.032 & 0.034 \\
\hline 0.50 & BIAS & 0.029 & -0.026 & 0.000 & -0.023 & 0.003 & 0.004 & 0.001 & 0.000 & -0.002 & 0.028 & -0.010 & -0.023 & -0.026 & 0.003 & 0.004 & 0.001 & 0.000 & -0.002 & 0.028 & 0.000 \\
\hline 0.50 & MSE & 0.025 & 0.031 & 0.030 & 0.030 & 0.027 & 0.029 & 0.030 & 0.030 & 0.030 & 0.028 & 0.027 & 0.030 & 0.030 & 0.027 & 0.029 & 0.030 & 0.030 & 0.030 & 0.028 & 0.030 \\
\hline 0.60 & BIAS & 0.019 & -0.026 & 0.000 & -0.023 & -0.002 & 0.002 & 0.000 & 0.000 & -0.002 & 0.022 & -0.014 & -0.023 & -0.025 & -0.002 & 0.002 & 0.000 & 0.000 & -0.002 & 0.022 & 0.000 \\
\hline 0.60 & MSE & 0.020 & 0.025 & 0.023 & 0.024 & 0.021 & 0.023 & 0.023 & 0.023 & 0.024 & 0.021 & 0.022 & 0.024 & 0.025 & 0.021 & 0.023 & 0.023 & 0.023 & 0.024 & 0.021 & 0.023 \\
\hline 0.70 & BIAS & 0.011 & -0.023 & 0.000 & -0.021 & -0.005 & 0.001 & 0.000 & 0.000 & -0.001 & 0.017 & -0.015 & -0.021 & -0.023 & -0.005 & 0.001 & 0.000 & 0.000 & -0.001 & 0.017 & 0.000 \\
\hline 0.70 & MSE & 0.014 & 0.017 & 0.016 & 0.017 & 0.015 & 0.015 & 0.016 & 0.016 & 0.016 & 0.014 & 0.016 & 0.017 & 0.017 & 0.015 & 0.015 & 0.016 & 0.016 & 0.016 & 0.014 & 0.016 \\
\hline 0.80 & BIAS & 0.005 & -0.018 & 0.000 & -0.017 & -0.006 & 0.000 & 0.000 & 0.000 & -0.001 & 0.011 & -0.014 & -0.017 & -0.018 & -0.006 & 0.000 & 0.000 & 0.000 & -0.001 & 0.011 & 0.000 \\
\hline 0.80 & MSE & 0.007 & 0.009 & 0.008 & 0.009 & 0.008 & 0.008 & 0.008 & 0.008 & 0.008 & 0.007 & 0.009 & 0.009 & 0.009 & 0.008 & 0.008 & 0.008 & 0.008 & 0.008 & 0.007 & 0.008 \\
\hline 0.90 & BIAS & 0.001 & -0.010 & 0.000 & -0.010 & -0.004 & 0.000 & 0.000 & 0.000 & 0.000 & 0.006 & -0.009 & -0.010 & -0.010 & -0.004 & 0.000 & 0.000 & 0.000 & 0.000 & 0.006 & 0.000 \\
\hline 0.90 & MSE & 0.002 & 0.003 & 0.002 & 0.003 & 0.002 & 0.002 & 0.002 & 0.002 & 0.002 & 0.002 & 0.003 & 0.003 & 0.003 & 0.002 & 0.002 & 0.002 & 0.002 & 0.002 & 0.002 & 0.002 \\
\hline
\end{tabular}


Table S6: Results for sample size $N=20$ and number of predictors $p=5$.

\begin{tabular}{|c|c|c|c|c|c|c|c|c|c|c|c|c|c|c|c|c|c|c|c|c|c|}
\hline$\rho^{2}$ & What & $R^{2}$ & $\mathrm{E}$ & OPE & $\mathrm{S}$ & W & OP1 & OP2 & OP5 & $\mathrm{P}$ & $\mathrm{C}$ & ML & $\mathrm{S}^{+}$ & $\mathrm{E}^{+}$ & $\mathrm{W}^{+}$ & $\mathrm{OP} 1^{+}$ & $\mathrm{OP}^{2+}$ & $\mathrm{OP}^{+}$ & $\mathrm{P}^{+}$ & $\mathrm{C}^{+}$ & $\mathrm{OPE}^{+}$ \\
\hline 0.00 & BIAS & 0.263 & 0.000 & 0.000 & 0.017 & 0.066 & 0.020 & 0.005 & 0.000 & -0.002 & 0.057 & 0.088 & 0.081 & 0.074 & 0.104 & 0.088 & 0.084 & 0.083 & 0.080 & 0.106 & 0.083 \\
\hline 0.00 & MSE & 0.087 & 0.034 & 0.041 & 0.033 & 0.034 & 0.038 & 0.040 & 0.041 & 0.040 & 0.039 & 0.024 & 0.022 & 0.020 & 0.029 & 0.025 & 0.024 & 0.023 & 0.023 & 0.031 & 0.023 \\
\hline 0.01 & BIAS & 0.260 & -0.001 & 0.000 & 0.016 & 0.065 & 0.019 & 0.005 & 0.000 & -0.002 & 0.056 & 0.084 & 0.077 & 0.070 & 0.101 & 0.084 & 0.079 & 0.078 & 0.076 & 0.102 & 0.078 \\
\hline 0.01 & MSE & 0.086 & 0.035 & 0.042 & 0.034 & 0.034 & 0.039 & 0.041 & 0.042 & 0.041 & 0.040 & 0.025 & 0.022 & 0.020 & 0.029 & 0.025 & 0.024 & 0.024 & 0.023 & 0.031 & 0.024 \\
\hline 0.05 & BIAS & 0.246 & -0.005 & 0.000 & 0.012 & 0.059 & 0.017 & 0.004 & 0.000 & -0.003 & 0.053 & 0.069 & 0.061 & 0.052 & 0.087 & 0.069 & 0.064 & 0.063 & 0.061 & 0.090 & 0.063 \\
\hline 0.05 & MSE & 0.082 & 0.038 & 0.046 & 0.037 & 0.037 & 0.042 & 0.045 & 0.046 & 0.045 & 0.043 & 0.027 & 0.024 & 0.022 & 0.030 & 0.027 & 0.027 & 0.026 & 0.026 & 0.033 & 0.026 \\
\hline 0.10 & BIAS & 0.230 & -0.009 & 0.000 & 0.007 & 0.052 & 0.015 & 0.004 & 0.000 & -0.003 & 0.050 & 0.052 & 0.043 & 0.034 & 0.072 & 0.054 & 0.049 & 0.047 & 0.044 & 0.076 & 0.047 \\
\hline 0.10 & MSE & 0.076 & 0.042 & 0.049 & 0.040 & 0.039 & 0.045 & 0.048 & 0.049 & 0.048 & 0.045 & 0.030 & 0.027 & 0.026 & 0.032 & 0.031 & 0.030 & 0.030 & 0.030 & 0.036 & 0.030 \\
\hline 0.20 & BIAS & 0.199 & -0.016 & 0.000 & -0.002 & 0.038 & 0.011 & 0.002 & 0.000 & -0.004 & 0.043 & 0.028 & 0.017 & 0.006 & 0.048 & 0.031 & 0.026 & 0.024 & 0.021 & 0.056 & 0.024 \\
\hline 0.20 & MSE & 0.064 & 0.046 & 0.052 & 0.044 & 0.041 & 0.048 & 0.051 & 0.052 & 0.052 & 0.048 & 0.035 & 0.034 & 0.033 & 0.036 & 0.037 & 0.038 & 0.038 & 0.037 & 0.041 & 0.038 \\
\hline 0.30 & BIAS & 0.169 & -0.021 & 0.000 & -0.009 & 0.027 & 0.008 & 0.002 & 0.000 & -0.004 & 0.037 & 0.011 & 0.000 & -0.011 & 0.031 & 0.017 & 0.012 & 0.011 & 0.007 & 0.043 & 0.011 \\
\hline 0.30 & MSE & 0.053 & 0.046 & 0.050 & 0.044 & 0.041 & 0.047 & 0.050 & 0.050 & 0.050 & 0.046 & 0.038 & 0.038 & 0.039 & 0.038 & 0.041 & 0.042 & 0.042 & 0.042 & 0.042 & 0.042 \\
\hline 0.40 & BIAS & 0.140 & -0.025 & 0.000 & -0.014 & 0.017 & 0.005 & 0.001 & 0.000 & -0.004 & 0.032 & 0.001 & -0.011 & -0.021 & 0.018 & 0.008 & 0.005 & 0.004 & 0.000 & 0.034 & 0.004 \\
\hline 0.40 & MSE & 0.043 & 0.043 & 0.045 & 0.041 & 0.037 & 0.043 & 0.045 & 0.045 & 0.045 & 0.041 & 0.038 & 0.039 & 0.040 & 0.036 & 0.040 & 0.041 & 0.041 & 0.041 & 0.039 & 0.041 \\
\hline 0.50 & BIAS & 0.112 & -0.026 & 0.000 & -0.017 & 0.009 & 0.003 & 0.000 & 0.000 & -0.003 & 0.026 & -0.007 & -0.016 & -0.025 & 0.009 & 0.004 & 0.002 & 0.001 & -0.002 & 0.027 & 0.001 \\
\hline 0.50 & MSE & 0.033 & 0.037 & 0.037 & 0.036 & 0.032 & 0.036 & 0.037 & 0.037 & 0.038 & 0.034 & 0.033 & 0.035 & 0.036 & 0.032 & 0.035 & 0.036 & 0.036 & 0.036 & 0.034 & 0.036 \\
\hline 0.60 & BIAS & 0.086 & -0.026 & 0.000 & -0.018 & 0.003 & 0.002 & 0.000 & 0.000 & -0.003 & 0.021 & -0.011 & -0.018 & -0.026 & 0.003 & 0.002 & 0.000 & 0.000 & -0.002 & 0.021 & 0.000 \\
\hline 0.60 & MSE & 0.023 & 0.029 & 0.028 & 0.028 & 0.025 & 0.027 & 0.028 & 0.028 & 0.028 & 0.026 & 0.026 & 0.028 & 0.029 & 0.025 & 0.027 & 0.028 & 0.028 & 0.028 & 0.025 & 0.028 \\
\hline 0.70 & BIAS & 0.062 & -0.023 & 0.000 & -0.017 & -0.002 & 0.001 & 0.000 & 0.000 & -0.002 & 0.016 & -0.013 & -0.017 & -0.023 & -0.002 & 0.001 & 0.000 & 0.000 & -0.002 & 0.016 & 0.000 \\
\hline 0.70 & MSE & 0.014 & 0.020 & 0.018 & 0.019 & 0.017 & 0.018 & 0.018 & 0.018 & 0.019 & 0.017 & 0.018 & 0.019 & 0.020 & 0.017 & 0.018 & 0.018 & 0.018 & 0.018 & 0.017 & 0.018 \\
\hline 0.80 & BIAS & 0.039 & -0.018 & 0.000 & -0.014 & -0.003 & 0.000 & 0.000 & 0.000 & -0.001 & 0.011 & -0.012 & -0.014 & -0.018 & -0.003 & 0.000 & 0.000 & 0.000 & -0.001 & 0.011 & 0.000 \\
\hline 0.80 & MSE & 0.007 & 0.011 & 0.009 & 0.010 & 0.009 & 0.009 & 0.009 & 0.009 & 0.009 & 0.008 & 0.010 & 0.010 & 0.011 & 0.009 & 0.009 & $\begin{array}{l}0.000 \\
0.009\end{array}$ & $\begin{array}{l}0.000 \\
0.009\end{array}$ & $\begin{array}{l}-0.001 \\
0.009\end{array}$ & $\begin{array}{l}.011 \\
0.008\end{array}$ & $\begin{array}{l}.0000 \\
0.009\end{array}$ \\
\hline 0.90 & BIAS & 0.019 & -0.010 & 0.000 & -0.008 & -0.003 & 0.000 & 0.000 & 0.000 & 0.000 & 0.006 & -0.008 & -0.008 & -0.010 & -0.003 & 0.000 & 0.000 & 0.000 & 0.000 & 0.006 & 0.000 \\
\hline 0.90 & MSE & 0.002 & 0.003 & 0.003 & 0.003 & 0.003 & 0.003 & 0.003 & 0.003 & 0.003 & 0.002 & 0.003 & 0.003 & 0.003 & 0.003 & 0.003 & 0.003 & 0.003 & 0.003 & 0.002 & 0.003 \\
\hline
\end{tabular}


Table S7: Results for sample size $N=20$ and number of predictors $p=10$.

\begin{tabular}{|c|c|c|c|c|c|c|c|c|c|c|c|c|c|c|c|c|c|c|c|c|c|}
\hline$\rho^{2}$ & What & $R^{2}$ & $\mathrm{E}$ & OPE & $\mathrm{S}$ & W & OP1 & OP2 & OP5 & $\mathrm{P}$ & C & ML & $\mathrm{S}^{+}$ & $\mathrm{E}^{+}$ & $\mathrm{w}^{+}$ & $\mathrm{OP} 1^{+}$ & $\mathrm{OP} 2^{+}$ & $\mathrm{OP}^{+}$ & $\mathrm{P}^{+}$ & $\mathrm{C}^{+}$ & $\mathrm{OPE}^{+}$ \\
\hline 0.00 & BIAS & 0.526 & -0.001 & -0.001 & 0.052 & 0.099 & 0.019 & 0.005 & 0.000 & -0.017 & 0.043 & 0.157 & 0.154 & 0.132 & 0.176 & 0.149 & 0.147 & 0.146 & 0.139 & 0.162 & 0.146 \\
\hline 0.00 & MSE & 0.300 & 0.105 & 0.129 & 0.097 & 0.095 & 0.117 & 0.125 & 0.129 & 0.132 & 0.119 & 0.063 & 0.061 & 0.052 & 0.071 & 0.061 & 0.060 & 0.060 & 0.057 & 0.068 & 0.060 \\
\hline 0.01 & BIAS & 0.520 & -0.001 & 0.000 & 0.051 & 0.098 & 0.019 & 0.005 & 0.000 & -0.017 & 0.042 & 0.152 & 0.148 & 0.127 & 0.171 & 0.144 & 0.141 & 0.141 & 0.134 & 0.158 & 0.141 \\
\hline 0.01 & MSE & 0.294 & 0.105 & 0.129 & 0.097 & 0.095 & 0.116 & 0.124 & 0.128 & 0.132 & 0.118 & 0.062 & 0.060 & 0.051 & 0.070 & 0.060 & 0.059 & 0.059 & 0.057 & 0.067 & 0.059 \\
\hline 0.05 & BIAS & 0.498 & -0.005 & 0.000 & 0.045 & 0.091 & 0.017 & 0.004 & 0.000 & -0.016 & 0.041 & 0.133 & 0.129 & 0.106 & 0.153 & 0.125 & 0.122 & 0.121 & 0.114 & 0.139 & 0.121 \\
\hline 0.05 & MSE & 0.271 & 0.104 & 0.125 & 0.095 & 0.092 & 0.114 & 0.122 & 0.125 & 0.129 & 0.115 & 0.060 & 0.058 & 0.050 & 0.067 & 0.059 & 0.058 & 0.058 & 0.056 & 0.065 & 0.058 \\
\hline 0.10 & BIAS & 0.470 & -0.009 & 0.000 & 0.039 & 0.082 & 0.015 & 0.004 & 0.000 & -0.015 & 0.039 & 0.111 & 0.106 & 0.082 & 0.131 & 0.103 & 0.099 & 0.099 & 0.091 & 0.118 & 0.099 \\
\hline 0.10 & MSE & 0.243 & 0.102 & 0.120 & 0.093 & 0.089 & 0.110 & 0.117 & 0.120 & 0.124 & 0.111 & 0.059 & 0.057 & 0.050 & 0.065 & 0.058 & 0.058 & 0.058 & 0.056 & 0.064 & 0.058 \\
\hline 0.20 & BIAS & 0.414 & -0.016 & 0.000 & 0.027 & 0.066 & 0.011 & 0.003 & 0.000 & -0.014 & 0.034 & 0.073 & 0.068 & 0.041 & 0.095 & 0.065 & 0.062 & 0.062 & 0.053 & 0.083 & 0.062 \\
\hline 0.20 & MSE & 0.192 & 0.095 & 0.108 & 0.086 & 0.081 & 0.100 & 0.106 & 0.108 & 0.112 & 0.100 & 0.058 & 0.057 & 0.053 & 0.061 & 0.059 & 0.059 & 0.059 & 0.058 & 0.063 & 0.059 \\
\hline 0.30 & BIAS & 0.358 & -0.021 & 0.000 & 0.017 & 0.051 & 0.008 & 0.002 & 0.000 & -0.012 & 0.030 & 0.045 & 0.039 & 0.011 & 0.066 & 0.038 & 0.035 & 0.034 & 0.026 & 0.056 & 0.034 \\
\hline 0.30 & MSE & 0.147 & 0.085 & 0.093 & 0.076 & 0.071 & 0.088 & 0.092 & 0.093 & 0.097 & 0.087 & 0.057 & 0.057 & 0.056 & 0.058 & 0.060 & 0.060 & 0.060 & 0.060 & 0.062 & 0.060 \\
\hline 0.40 & BIAS & 0.304 & -0.025 & 0.000 & 0.008 & 0.038 & 0.005 & 0.001 & 0.000 & -0.010 & 0.026 & 0.024 & 0.019 & -0.009 & 0.045 & 0.020 & 0.017 & 0.017 & 0.009 & 0.038 & 0.017 \\
\hline 0.40 & MSE & 0.109 & 0.073 & 0.077 & 0.065 & 0.060 & 0.073 & 0.076 & 0.077 & 0.080 & 0.072 & 0.054 & 0.054 & 0.056 & 0.053 & 0.057 & 0.058 & 0.058 & 0.059 & 0.058 & 0.058 \\
\hline 0.50 & BIAS & 0.251 & -0.026 & 0.000 & 0.001 & 0.026 & 0.003 & 0.000 & 0.000 & -0.008 & 0.022 & 0.011 & 0.005 & -0.020 & 0.029 & 0.009 & 0.007 & 0.006 & 0.000 & 0.027 & 0.006 \\
\hline 0.50 & MSE & 0.076 & 0.059 & 0.060 & 0.052 & 0.048 & 0.057 & 0.059 & 0.060 & 0.062 & 0.056 & 0.047 & 0.047 & 0.051 & 0.045 & 0.050 & 0.051 & 0.051 & 0.052 & 0.049 & 0.051 \\
\hline 0.60 & BIAS & 0.198 & -0.026 & 0.000 & -0.004 & 0.017 & 0.001 & 0.000 & 0.000 & -0.006 & 0.018 & 0.002 & -0.002 & -0.024 & 0.017 & 0.003 & 0.002 & 0.002 & -0.004 & 0.019 & 0.002 \\
\hline 0.60 & MSE & 0.049 & 0.044 & 0.042 & 0.039 & 0.035 & 0.041 & 0.042 & 0.042 & 0.044 & 0.039 & 0.036 & 0.037 & 0.041 & 0.034 & 0.039 & 0.039 & 0.040 & 0.041 & 0.037 & 0.040 \\
\hline 0.70 & BIAS & 0.147 & -0.023 & 0.000 & -0.006 & 0.009 & 0.000 & 0.000 & 0.000 & -0.004 & 0.014 & -0.003 & -0.006 & -0.023 & 0.009 & 0.001 & 0.000 & 0.000 & -0.004 & 0.014 & 0.000 \\
\hline 0.70 & MSE & 0.028 & 0.029 & 0.026 & 0.025 & 0.023 & 0.026 & 0.026 & 0.026 & 0.027 & 0.024 & 0.024 & 0.025 & 0.028 & 0.023 & 0.025 & 0.026 & 0.026 & 0.027 & 0.024 & 0.026 \\
\hline 0.80 & BIAS & 0.097 & -0.018 & 0.000 & -0.007 & 0.004 & 0.000 & 0.000 & 0.000 & -0.002 & 0.010 & -0.005 & -0.007 & -0.018 & 0.004 & 0.000 & 0.000 & 0.000 & -0.002 & 0.010 & 0.000 \\
\hline 0.80 & MSE & 0.013 & 0.015 & 0.013 & 0.013 & 0.012 & 0.013 & 0.013 & 0.013 & 0.013 & 0.012 & 0.013 & 0.013 & 0.015 & 0.012 & 0.013 & 0.013 & 0.013 & 0.013 & 0.012 & 0.013 \\
\hline 0.90 & BIAS & & -0.011 & 0.000 & & & 0.000 & & & & & & & & & 0.000 & & & -0.001 & 0.005 & 0.000 \\
\hline 0.90 & MSE & 0.003 & 0.004 & 0.004 & 0.004 & 0.003 & 0.004 & 0.004 & 0.004 & 0.004 & 0.003 & 0.004 & 0.004 & 0.004 & 0.003 & 0.004 & 0.004 & 0.004 & 0.004 & 0.003 & 0.004 \\
\hline
\end{tabular}


Table S8: Results for sample size $N=30$ and number of predictors $p=2$.

\begin{tabular}{|c|c|c|c|c|c|c|c|c|c|c|c|c|c|c|c|c|c|c|c|c|c|}
\hline$\rho^{2}$ & What & $R^{2}$ & $\mathrm{E}$ & OPE & $\mathrm{S}$ & W & OP1 & OP2 & OP5 & $\mathrm{P}$ & C & ML & $\mathrm{S}^{+}$ & $\mathrm{E}^{+}$ & $\mathrm{w}^{+}$ & $\mathrm{OP} 1^{+}$ & $\mathrm{OP} 2^{+}$ & $\mathrm{OP}^{+}$ & $\mathrm{P}^{+}$ & $\mathrm{C}^{+}$ & $\mathrm{OPE}^{+}$ \\
\hline 0.00 & BIAS & 0.069 & 0.000 & 0.000 & 0.003 & 0.036 & 0.009 & 0.002 & 0.000 & 0.001 & 0.038 & 0.034 & 0.027 & 0.026 & 0.043 & 0.031 & 0.029 & 0.028 & 0.028 & 0.046 & 0.028 \\
\hline 0.00 & MSE & 0.009 & 0.005 & 0.006 & 0.005 & 0.006 & 0.005 & 0.005 & 0.006 & 0.005 & 0.006 & 0.005 & 0.004 & 0.003 & 0.006 & 0.004 & 0.004 & 0.004 & 0.004 & 0.006 & 0.004 \\
\hline 0.01 & BIAS & 0.068 & 0.000 & 0.000 & 0.002 & 0.035 & 0.009 & 0.002 & 0.000 & 0.001 & 0.038 & 0.031 & 0.024 & 0.023 & 0.041 & 0.029 & 0.026 & 0.026 & 0.026 & 0.045 & 0.026 \\
\hline 0.01 & MSE & 0.010 & 0.006 & 0.007 & 0.006 & 0.007 & 0.006 & 0.007 & 0.007 & 0.007 & 0.007 & 0.006 & 0.004 & 0.004 & 0.006 & 0.005 & 0.005 & 0.005 & 0.005 & 0.007 & 0.005 \\
\hline 0.05 & BIAS & 0.063 & -0.003 & 0.000 & -0.001 & 0.031 & 0.008 & 0.002 & 0.000 & 0.001 & 0.036 & 0.024 & 0.014 & 0.012 & 0.035 & 0.021 & 0.017 & 0.017 & 0.017 & 0.040 & 0.017 \\
\hline 0.05 & MSE & 0.012 & 0.010 & 0.011 & 0.010 & 0.010 & 0.010 & 0.011 & 0.011 & 0.011 & 0.011 & 0.009 & 0.007 & 0.007 & 0.009 & 0.009 & 0.008 & 0.008 & 0.008 & 0.011 & 0.008 \\
\hline 0.10 & BIAS & 0.057 & -0.006 & 0.000 & -0.003 & 0.027 & 0.007 & 0.001 & 0.000 & 0.001 & 0.034 & 0.018 & 0.005 & 0.003 & 0.029 & 0.014 & 0.010 & 0.009 & 0.009 & 0.036 & 0.009 \\
\hline 0.10 & MSE & 0.015 & 0.014 & 0.015 & 0.013 & 0.013 & 0.014 & 0.015 & 0.015 & 0.015 & 0.015 & 0.013 & 0.012 & 0.011 & 0.013 & 0.013 & 0.013 & 0.013 & 0.013 & 0.014 & 0.013 \\
\hline 0.20 & BIAS & 0.046 & -0.010 & 0.000 & -0.008 & 0.019 & 0.005 & 0.001 & 0.000 & 0.000 & 0.030 & 0.010 & -0.006 & -0.008 & 0.019 & 0.007 & 0.003 & 0.003 & 0.002 & 0.030 & 0.003 \\
\hline 0.20 & MSE & 0.018 & 0.019 & 0.020 & 0.019 & 0.018 & 0.020 & 0.020 & 0.020 & 0.020 & 0.020 & 0.018 & 0.018 & 0.018 & 0.018 & 0.019 & 0.019 & 0.019 & 0.019 & 0.020 & 0.019 \\
\hline 0.30 & BIAS & 0.036 & -0.014 & 0.000 & -0.012 & 0.012 & 0.004 & 0.001 & 0.000 & 0.000 & 0.026 & 0.003 & -0.012 & -0.013 & 0.012 & 0.004 & 0.001 & 0.001 & 0.000 & 0.026 & 0.001 \\
\hline 0.30 & MSE & 0.020 & 0.021 & 0.022 & 0.021 & 0.020 & 0.022 & 0.022 & 0.022 & 0.022 & 0.021 & 0.020 & 0.021 & 0.021 & 0.020 & 0.022 & 0.022 & 0.022 & 0.022 & 0.021 & 0.022 \\
\hline 0.40 & BIAS & 0.027 & -0.016 & 0.000 & -0.014 & 0.006 & 0.003 & 0.001 & 0.000 & 0.000 & 0.022 & -0.002 & -0.014 & -0.016 & 0.006 & 0.003 & 0.001 & 0.000 & 0.000 & 0.022 & 0.000 \\
\hline 0.40 & MSE & 0.019 & 0.021 & 0.022 & 0.021 & 0.020 & 0.021 & 0.022 & 0.022 & 0.022 & 0.021 & 0.020 & 0.021 & 0.021 & 0.020 & 0.021 & 0.022 & 0.022 & 0.022 & 0.021 & 0.022 \\
\hline 0.50 & BIAS & 0.019 & -0.017 & 0.000 & -0.016 & 0.002 & 0.002 & 0.001 & 0.000 & 0.000 & 0.018 & -0.007 & -0.016 & -0.017 & 0.002 & 0.002 & 0.001 & 0.000 & 0.000 & 0.018 & 0.000 \\
\hline 0.50 & MSE & 0.017 & 0.019 & 0.019 & 0.019 & 0.017 & 0.018 & 0.019 & 0.019 & 0.019 & 0.018 & 0.017 & 0.019 & 0.019 & 0.017 & 0.018 & 0.019 & 0.019 & 0.019 & 0.018 & 0.019 \\
\hline 0.60 & BIAS & 0.012 & -0.016 & 0.000 & -0.015 & -0.001 & 0.001 & 0.000 & 0.000 & 0.000 & 0.015 & -0.009 & -0.015 & -0.016 & -0.001 & 0.001 & 0.000 & 0.000 & 0.000 & 0.015 & 0.000 \\
\hline 0.60 & MSE & 0.013 & 0.015 & 0.014 & 0.015 & 0.014 & 0.014 & 0.014 & 0.014 & 0.015 & 0.014 & 0.014 & 0.015 & 0.015 & 0.014 & 0.014 & 0.014 & 0.014 & 0.015 & 0.014 & 0.014 \\
\hline 0.70 & BIAS & 0.007 & -0.015 & 0.000 & -0.014 & -0.003 & 0.001 & 0.000 & 0.000 & 0.000 & 0.011 & -0.010 & -0.014 & -0.015 & -0.003 & 0.001 & 0.000 & 0.000 & 0.000 & 0.011 & 0.000 \\
\hline 0.70 & MSE & 0.009 & 0.010 & 0.010 & 0.010 & 0.009 & 0.010 & 0.010 & 0.010 & 0.010 & 0.009 & 0.010 & 0.010 & 0.010 & 0.009 & 0.010 & 0.010 & 0.010 & 0.010 & 0.009 & 0.010 \\
\hline 0.80 & BIAS & 0.003 & -0.011 & 0.000 & -0.011 & -0.004 & 0.000 & 0.000 & 0.000 & 0.000 & 0.007 & -0.009 & -0.011 & -0.011 & -0.004 & 0.000 & 0.000 & 0.000 & 0.000 & 0.007 & 0.000 \\
\hline 0.80 & MSE & 0.005 & 0.005 & 0.005 & 0.005 & 0.005 & 0.005 & 0.005 & 0.005 & 0.005 & 0.005 & 0.005 & 0.005 & 0.005 & 0.005 & 0.005 & 0.005 & 0.005 & 0.005 & 0.005 & 0.005 \\
\hline 0.90 & BIAS & 0.001 & -0.006 & 0.000 & -0.006 & -0.003 & 0.000 & 0.000 & 0.000 & 0.000 & 0.004 & -0.006 & -0.006 & -0.006 & -0.003 & 0.000 & 0.000 & 0.000 & 0.000 & 0.004 & 0.000 \\
\hline 0.90 & MSE & 0.001 & 0.002 & 0.001 & 0.002 & 0.001 & 0.001 & 0.001 & 0.001 & 0.001 & 0.001 & 0.002 & 0.002 & 0.002 & 0.001 & 0.001 & 0.001 & 0.001 & 0.001 & 0.001 & 0.001 \\
\hline
\end{tabular}


Table S9: Results for sample size $N=30$ and number of predictors $p=5$.

\begin{tabular}{|c|c|c|c|c|c|c|c|c|c|c|c|c|c|c|c|c|c|c|c|c|c|}
\hline$\rho^{2}$ & What & $R^{2}$ & $\mathrm{E}$ & OPE & $\mathrm{S}$ & $\mathrm{W}$ & OP1 & OP2 & OP5 & P & C & ML & $\mathrm{S}^{+}$ & $\mathrm{E}^{+}$ & $\mathrm{w}^{+}$ & $\mathrm{OP} 1^{+}$ & $\mathrm{OP} 2^{+}$ & $\mathrm{OP}^{+}$ & $\mathrm{P}^{+}$ & $\mathrm{C}^{+}$ & $\mathrm{OPE}^{+}$ \\
\hline 0.00 & BIAS & 0.173 & 0.000 & 0.000 & 0.007 & 0.040 & 0.009 & 0.002 & 0.000 & 0.000 & 0.037 & 0.054 & 0.049 & 0.046 & 0.065 & 0.053 & 0.050 & 0.050 & 0.049 & 0.066 & 0.050 \\
\hline 0.00 & MSE & 0.039 & 0.014 & 0.015 & 0.013 & 0.014 & 0.015 & 0.015 & 0.015 & 0.015 & 0.016 & 0.010 & 0.009 & 0.008 & 0.012 & 0.010 & 0.010 & 0.009 & 0.009 & 0.013 & 0.009 \\
\hline 0.01 & BIAS & 0.170 & -0.001 & 0.000 & 0.006 & 0.039 & 0.009 & 0.002 & 0.000 & 0.000 & 0.037 & 0.051 & 0.045 & 0.042 & 0.062 & 0.049 & 0.046 & 0.046 & 0.046 & 0.063 & 0.046 \\
\hline 0.01 & MSE & 0.039 & 0.014 & 0.016 & 0.014 & 0.015 & 0.016 & 0.016 & 0.016 & 0.016 & 0.016 & 0.011 & 0.009 & 0.009 & 0.013 & 0.010 & 0.010 & 0.010 & 0.010 & 0.013 & 0.010 \\
\hline 0.05 & BIAS & 0.161 & -0.003 & 0.000 & 0.003 & 0.035 & 0.008 & 0.001 & 0.000 & 0.000 & 0.035 & 0.038 & 0.031 & 0.028 & 0.051 & 0.036 & 0.033 & 0.033 & 0.032 & 0.053 & 0.033 \\
\hline 0.05 & MSE & 0.038 & 0.018 & 0.020 & 0.017 & 0.017 & 0.019 & 0.020 & 0.020 & 0.020 & 0.019 & 0.013 & 0.012 & 0.011 & 0.015 & 0.013 & 0.013 & 0.013 & 0.013 & 0.016 & 0.013 \\
\hline 0.10 & BIAS & 0.150 & -0.006 & 0.000 & 0.000 & 0.030 & 0.007 & 0.001 & 0.000 & -0.001 & 0.033 & 0.027 & 0.018 & 0.014 & 0.040 & 0.025 & 0.021 & 0.021 & 0.020 & 0.044 & 0.021 \\
\hline 0.10 & MSE & 0.037 & 0.021 & 0.023 & 0.021 & 0.020 & 0.022 & 0.023 & 0.023 & 0.023 & 0.022 & 0.017 & 0.015 & 0.015 & 0.018 & 0.017 & 0.017 & 0.017 & 0.017 & 0.019 & 0.017 \\
\hline 0.20 & BIAS & 0.129 & -0.011 & 0.000 & -0.005 & 0.022 & 0.005 & 0.001 & 0.000 & -0.001 & 0.028 & 0.012 & 0.001 & -0.004 & 0.025 & 0.011 & 0.008 & 0.007 & 0.006 & 0.032 & 0.007 \\
\hline 0.20 & MSE & 0.034 & 0.025 & 0.027 & 0.025 & 0.023 & 0.026 & 0.027 & 0.027 & 0.027 & 0.026 & 0.022 & 0.022 & 0.022 & 0.022 & 0.023 & 0.023 & 0.023 & 0.023 & 0.024 & 0.023 \\
\hline 0.30 & BIAS & 0.109 & -0.014 & 0.000 & -0.009 & 0.014 & 0.003 & 0.000 & 0.000 & -0.001 & 0.024 & 0.003 & -0.008 & -0.012 & 0.015 & 0.005 & 0.002 & 0.002 & 0.001 & 0.025 & 0.002 \\
\hline 0.30 & MSE & 0.030 & 0.026 & 0.028 & 0.026 & 0.024 & 0.027 & 0.028 & 0.028 & 0.028 & 0.026 & 0.024 & 0.025 & 0.025 & 0.024 & 0.026 & 0.026 & 0.026 & 0.026 & 0.026 & 0.026 \\
\hline 0.40 & BIAS & 0.090 & -0.017 & 0.000 & -0.012 & 0.008 & 0.002 & 0.000 & 0.000 & -0.002 & 0.021 & -0.002 & -0.012 & -0.016 & 0.008 & 0.002 & 0.000 & 0.000 & -0.001 & 0.021 & 0.000 \\
\hline 0.40 & MSE & 0.025 & 0.025 & 0.026 & 0.025 & 0.023 & 0.025 & 0.025 & 0.026 & 0.026 & 0.024 & 0.023 & 0.024 & 0.025 & 0.023 & 0.025 & 0.025 & 0.025 & 0.025 & 0.024 & 0.025 \\
\hline 0.50 & BIAS & 0.072 & -0.017 & 0.000 & -0.014 & 0.003 & 0.001 & 0.000 & 0.000 & -0.001 & 0.017 & -0.006 & -0.014 & -0.017 & 0.003 & 0.001 & 0.000 & 0.000 & -0.001 & 0.017 & 0.000 \\
\hline 0.50 & MSE & 0.020 & 0.022 & 0.022 & 0.021 & 0.020 & 0.021 & 0.021 & 0.022 & 0.022 & 0.020 & 0.020 & 0.021 & 0.022 & 0.020 & 0.021 & 0.021 & 0.021 & 0.022 & 0.020 & 0.021 \\
\hline 0.60 & BIAS & 0.055 & -0.017 & 0.000 & -0.014 & 0.000 & 0.001 & 0.000 & 0.000 & -0.001 & 0.014 & -0.009 & -0.014 & -0.017 & 0.000 & 0.001 & 0.000 & 0.000 & -0.001 & 0.014 & 0.000 \\
\hline 0.60 & MSE & 0.014 & 0.017 & 0.016 & 0.017 & 0.015 & 0.016 & 0.016 & 0.016 & 0.016 & 0.015 & 0.016 & 0.017 & 0.017 & 0.015 & 0.016 & 0.016 & 0.016 & 0.016 & 0.015 & 0.016 \\
\hline 0.70 & BIAS & 0.039 & -0.015 & 0.000 & -0.013 & -0.002 & 0.000 & 0.000 & 0.000 & -0.001 & 0.010 & -0.010 & -0.013 & -0.015 & -0.002 & 0.000 & 0.000 & 0.000 & -0.001 & 0.010 & 0.000 \\
\hline 0.70 & MSE & 0.009 & 0.011 & 0.011 & 0.011 & 0.010 & 0.011 & 0.011 & 0.011 & 0.011 & 0.010 & 0.011 & 0.011 & 0.011 & 0.010 & 0.011 & 0.011 & 0.011 & 0.011 & 0.010 & 0.011 \\
\hline 0.80 & BIAS & 0.025 & -0.012 & 0.000 & -0.010 & -0.003 & 0.000 & 0.000 & 0.000 & 0.000 & 0.007 & -0.009 & -0.010 & -0.012 & -0.003 & 0.000 & 0.000 & 0.000 & 0.000 & 0.007 & 0.000 \\
\hline 0.80 & MSE & 0.005 & 0.006 & 0.005 & 0.006 & 0.005 & 0.005 & 0.005 & 0.005 & 0.005 & 0.005 & 0.006 & 0.006 & 0.006 & 0.005 & 0.005 & 0.005 & 0.005 & 0.005 & 0.005 & 0.005 \\
\hline 0.90 & BIAS & 0.012 & -0.007 & 0.000 & -0.006 & -0.002 & 0.000 & 0.000 & 0.000 & 0.000 & 0.004 & -0.005 & -0.006 & -0.007 & -0.002 & 0.000 & 0.00 & 0.0 & 0.000 & 0.004 & 0.000 \\
\hline 0.90 & MSE & 0.001 & 0.002 & 0.002 & 0.002 & 0.002 & 0.002 & 0.002 & 0.002 & 0.002 & 0.001 & 0.002 & 0.002 & 0.002 & 0.002 & 0.002 & 0.002 & 0.002 & 0.002 & 0.001 & 0.002 \\
\hline
\end{tabular}


Table S10: Results for sample size $N=30$ and number of predictors $p=10$.

\begin{tabular}{|c|c|c|c|c|c|c|c|c|c|c|c|c|c|c|c|c|c|c|c|c|c|}
\hline$\rho^{2}$ & What & $R^{2}$ & $\mathrm{E}$ & OPE & $\mathrm{S}$ & $\mathrm{W}$ & OP1 & OP2 & OP5 & $\mathrm{P}$ & C & ML & $\mathrm{S}^{+}$ & $\mathrm{E}^{+}$ & $\mathrm{W}^{+}$ & $\mathrm{OP} 1^{+}$ & $\mathrm{OP} 2^{+}$ & $\mathrm{OP}^{+}$ & $\mathrm{P}^{+}$ & $\mathrm{C}^{+}$ & $\mathrm{OPE}^{+}$ \\
\hline 0.00 & BIAS & 0.345 & 0.000 & 0.000 & 0.017 & 0.050 & 0.009 & 0.002 & 0.000 & -0.002 & 0.035 & 0.085 & 0.082 & 0.074 & 0.097 & 0.082 & 0.080 & 0.080 & 0.078 & 0.094 & 0.080 \\
\hline 0.00 & MSE & 0.133 & 0.034 & 0.039 & 0.033 & 0.033 & 0.037 & 0.038 & 0.039 & 0.039 & 0.037 & 0.022 & 0.021 & 0.018 & 0.025 & 0.021 & 0.021 & 0.021 & 0.020 & 0.025 & 0.021 \\
\hline 0.01 & BIAS & 0.341 & -0.001 & 0.000 & 0.016 & 0.049 & 0.009 & 0.001 & 0.000 & -0.002 & 0.034 & 0.081 & 0.077 & 0.070 & 0.093 & 0.078 & 0.076 & 0.075 & 0.074 & 0.091 & 0.075 \\
\hline 0.01 & MSE & 0.131 & 0.035 & 0.039 & 0.034 & 0.034 & 0.038 & 0.039 & 0.039 & 0.039 & 0.037 & 0.022 & 0.021 & 0.019 & 0.025 & 0.021 & 0.021 & 0.021 & 0.020 & 0.025 & 0.021 \\
\hline 0.05 & BIAS & 0.325 & -0.003 & 0.000 & 0.013 & 0.044 & 0.008 & 0.001 & 0.000 & -0.003 & 0.033 & 0.065 & 0.060 & 0.052 & 0.078 & 0.062 & 0.059 & 0.059 & 0.057 & 0.076 & 0.059 \\
\hline 0.05 & MSE & 0.121 & 0.036 & 0.041 & 0.035 & 0.035 & 0.039 & 0.041 & 0.041 & 0.041 & 0.039 & 0.023 & 0.022 & 0.020 & 0.026 & 0.023 & 0.022 & 0.022 & 0.022 & 0.026 & 0.022 \\
\hline 0.10 & BIAS & 0.306 & -0.006 & 0.000 & 0.009 & 0.039 & 0.006 & 0.001 & 0.000 & -0.003 & 0.031 & 0.048 & 0.043 & 0.033 & 0.063 & 0.045 & 0.042 & 0.042 & 0.040 & 0.061 & 0.042 \\
\hline 0.10 & MSE & 0.110 & 0.038 & 0.042 & 0.037 & 0.036 & 0.041 & 0.042 & 0.042 & 0.042 & 0.040 & 0.025 & 0.024 & 0.023 & 0.027 & 0.025 & 0.025 & 0.025 & 0.025 & 0.028 & 0.025 \\
\hline 0.20 & BIAS & 0.269 & -0.011 & 0.000 & 0.003 & 0.030 & 0.004 & 0.000 & 0.000 & -0.003 & 0.027 & 0.024 & 0.018 & 0.007 & 0.039 & 0.022 & 0.019 & 0.019 & 0.017 & 0.040 & 0.019 \\
\hline 0.20 & MSE & 0.089 & 0.039 & 0.042 & 0.037 & 0.036 & 0.041 & 0.042 & 0.042 & 0.042 & 0.040 & 0.030 & 0.029 & 0.029 & 0.030 & 0.031 & 0.031 & 0.031 & 0.031 & 0.032 & 0.031 \\
\hline 0.30 & BIAS & 0.232 & -0.015 & 0.000 & -0.002 & 0.021 & 0.003 & 0.000 & 0.000 & -0.003 & 0.023 & 0.010 & 0.003 & -0.008 & 0.025 & 0.009 & 0.007 & 0.007 & 0.004 & 0.028 & 0.007 \\
\hline 0.30 & MSE & 0.070 & 0.037 & 0.039 & 0.036 & 0.034 & 0.038 & 0.039 & 0.039 & 0.039 & 0.037 & 0.032 & 0.032 & 0.032 & 0.031 & 0.033 & 0.034 & 0.034 & 0.034 & 0.034 & 0.034 \\
\hline 0.40 & BIAS & 0.196 & -0.017 & 0.000 & -0.006 & 0.014 & 0.002 & 0.000 & 0.000 & -0.003 & 0.020 & 0.002 & -0.005 & -0.015 & 0.015 & 0.004 & 0.002 & 0.002 & -0.001 & 0.021 & 0.002 \\
\hline 0.40 & MSE & 0.052 & 0.033 & 0.034 & 0.032 & 0.030 & 0.033 & 0.034 & 0.034 & 0.034 & 0.032 & 0.030 & 0.030 & 0.031 & 0.029 & 0.032 & 0.032 & 0.032 & 0.032 & 0.031 & 0.032 \\
\hline 0.50 & BIAS & 0.161 & -0.018 & 0.000 & -0.009 & 0.008 & 0.001 & 0.000 & 0.000 & -0.002 & 0.016 & -0.003 & -0.008 & -0.017 & 0.008 & 0.001 & 0.000 & 0.000 & -0.002 & 0.017 & 0.000 \\
\hline 0.50 & MSE & 0.038 & 0.028 & 0.027 & 0.026 & 0.025 & 0.027 & 0.027 & 0.027 & 0.028 & 0.026 & 0.025 & 0.026 & 0.027 & 0.024 & 0.027 & 0.027 & 0.027 & 0.027 & 0.026 & 0.027 \\
\hline 0.60 & BIAS & 0.127 & -0.017 & 0.000 & -0.010 & 0.004 & 0.000 & 0.000 & 0.000 & -0.002 & 0.013 & -0.006 & -0.010 & -0.017 & 0.004 & 0.000 & 0.000 & 0.000 & -0.002 & 0.013 & 0.000 \\
\hline 0.60 & MSE & 0.025 & 0.021 & 0.020 & 0.020 & 0.018 & 0.020 & 0.020 & 0.020 & 0.020 & 0.019 & 0.019 & 0.020 & 0.021 & 0.018 & 0.020 & 0.020 & 0.020 & 0.020 & 0.019 & 0.020 \\
\hline 0.70 & BIAS & 0.094 & -0.015 & 0.000 & -0.010 & 0.001 & 0.000 & 0.000 & 0.000 & -0.001 & 0.010 & -0.007 & -0.010 & -0.015 & 0.001 & 0.000 & 0.000 & 0.000 & -0.001 & 0.010 & 0.000 \\
\hline 0.70 & MSE & 0.014 & 0.014 & 0.013 & 0.013 & 0.012 & 0.013 & 0.013 & 0.013 & 0.013 & 0.012 & 0.013 & 0.013 & 0.014 & 0.012 & 0.013 & 0.013 & 0.013 & 0.013 & 0.012 & 0.013 \\
\hline 0.80 & BIAS & 0.061 & -0.012 & 0.000 & -0.008 & -0.001 & 0.000 & 0.000 & 0.000 & -0.001 & 0.007 & -0.007 & -0.008 & -0.012 & -0.001 & 0.000 & 0.000 & 0.000 & -0.001 & 0.007 & 0.000 \\
\hline 0.80 & MSE & 0.007 & 0.007 & 0.006 & 0.007 & 0.006 & 0.006 & 0.006 & 0.006 & 0.006 & 0.006 & 0.007 & 0.007 & 0.007 & 0.006 & 0.006 & 0.006 & 0.006 & 0.006 & 0.006 & 0.006 \\
\hline 0.90 & BIAS & 0.030 & -0.007 & 0.000 & -0.005 & -0.001 & 0.000 & 0.000 & 0.000 & 0.000 & 0.004 & -0.004 & -0.005 & -0.007 & -0.001 & 0.000 & 0.000 & 0.000 & 0.000 & 0.004 & 0.000 \\
\hline 0.90 & MSE & 0.002 & 0.002 & 0.002 & 0.002 & 0.002 & 0.002 & 0.002 & 0.002 & 0.002 & 0.002 & 0.002 & 0.002 & 0.002 & 0.002 & 0.002 & 0.002 & 0.002 & 0.002 & 0.002 & 0.002 \\
\hline
\end{tabular}


Table S11: Results for sample size $N=40$ and number of predictors $p=2$.

\begin{tabular}{|c|c|c|c|c|c|c|c|c|c|c|c|c|c|c|c|c|c|c|c|c|c|}
\hline$\rho^{2}$ & What & $R^{2}$ & $\mathrm{E}$ & OPE & $\mathrm{S}$ & $\mathrm{W}$ & OP1 & OP2 & OP5 & P & C & ML & $\mathrm{S}^{+}$ & $\mathrm{E}^{+}$ & $\mathrm{w}^{+}$ & $\mathrm{OP} 1^{+}$ & $\mathrm{OP} 2^{+}$ & $\mathrm{OP}^{+}$ & $\mathrm{P}^{+}$ & $\mathrm{C}^{+}$ & $\mathrm{OPE}^{+}$ \\
\hline 0.00 & BIAS & 0.051 & 0.000 & 0.000 & 0.001 & 0.026 & 0.005 & 0.001 & 0.000 & 0.001 & 0.028 & 0.025 & 0.020 & 0.019 & 0.032 & 0.022 & 0.020 & 0.020 & 0.020 & 0.033 & 0.020 \\
\hline 0.00 & MSE & 0.005 & 0.003 & 0.003 & 0.003 & 0.003 & 0.003 & 0.003 & 0.003 & 0.003 & 0.003 & 0.003 & 0.002 & 0.002 & 0.003 & 0.002 & 0.002 & 0.002 & 0.002 & 0.003 & 0.002 \\
\hline 0.01 & BIAS & 0.050 & -0.001 & 0.000 & 0.001 & 0.025 & 0.005 & 0.000 & 0.000 & 0.000 & 0.027 & 0.023 & 0.017 & 0.016 & 0.030 & 0.019 & 0.018 & 0.018 & 0.018 & 0.032 & 0.018 \\
\hline 0.01 & MSE & 0.006 & 0.003 & 0.004 & 0.003 & 0.004 & 0.004 & 0.004 & 0.004 & 0.004 & 0.004 & 0.003 & 0.003 & 0.002 & 0.004 & 0.003 & 0.003 & 0.003 & 0.003 & 0.004 & 0.003 \\
\hline 0.05 & BIAS & 0.046 & -0.003 & 0.000 & -0.001 & 0.022 & 0.004 & 0.000 & 0.000 & 0.000 & 0.026 & 0.017 & 0.008 & 0.007 & 0.025 & 0.012 & 0.010 & 0.010 & 0.010 & 0.028 & 0.010 \\
\hline 0.05 & MSE & 0.008 & 0.006 & 0.007 & 0.006 & 0.007 & 0.007 & 0.007 & 0.007 & 0.007 & 0.007 & 0.006 & 0.005 & 0.005 & 0.006 & 0.006 & 0.006 & 0.006 & 0.006 & 0.007 & 0.006 \\
\hline 0.10 & BIAS & 0.042 & -0.005 & 0.000 & -0.004 & 0.019 & 0.003 & 0.000 & 0.000 & 0.000 & 0.024 & 0.013 & 0.000 & -0.001 & 0.020 & 0.007 & 0.004 & 0.004 & 0.004 & 0.025 & 0.004 \\
\hline 0.10 & MSE & 0.010 & 0.010 & 0.010 & 0.010 & 0.009 & 0.010 & 0.010 & 0.010 & 0.010 & 0.010 & 0.009 & 0.009 & 0.009 & 0.009 & 0.009 & 0.009 & 0.009 & 0.009 & 0.010 & 0.009 \\
\hline 0.20 & BIAS & 0.033 & -0.008 & 0.000 & -0.007 & 0.013 & 0.002 & 0.000 & 0.000 & 0.000 & 0.021 & 0.007 & -0.007 & -0.008 & 0.013 & 0.003 & 0.001 & 0.000 & 0.000 & 0.021 & 0.000 \\
\hline 0.20 & MSE & 0.014 & 0.014 & 0.015 & 0.014 & 0.013 & 0.014 & 0.015 & 0.015 & 0.015 & 0.014 & 0.013 & 0.014 & 0.014 & 0.013 & 0.014 & 0.014 & 0.014 & 0.014 & 0.014 & 0.014 \\
\hline 0.30 & BIAS & 0.026 & -0.011 & 0.000 & -0.010 & 0.008 & 0.002 & 0.000 & 0.000 & -0.001 & 0.018 & 0.002 & -0.010 & -0.011 & 0.008 & 0.002 & 0.000 & 0.000 & 0.000 & 0.018 & 0.000 \\
\hline 0.30 & MSE & 0.015 & 0.016 & 0.016 & 0.016 & 0.015 & 0.016 & 0.016 & 0.016 & 0.016 & 0.016 & 0.015 & 0.016 & 0.016 & 0.015 & 0.016 & 0.016 & 0.016 & 0.016 & 0.016 & 0.016 \\
\hline 0.40 & BIAS & 0.019 & -0.012 & 0.000 & -0.011 & 0.004 & 0.001 & 0.000 & 0.000 & -0.001 & 0.016 & -0.002 & -0.011 & -0.012 & 0.004 & 0.001 & 0.000 & 0.000 & -0.001 & 0.016 & 0.000 \\
\hline 0.40 & MSE & 0.014 & 0.016 & 0.016 & 0.016 & 0.015 & 0.016 & 0.016 & 0.016 & 0.016 & 0.015 & 0.015 & 0.016 & 0.016 & 0.015 & 0.016 & 0.016 & 0.016 & 0.016 & 0.015 & 0.016 \\
\hline 0.50 & BIAS & 0.013 & -0.013 & 0.000 & -0.012 & 0.001 & 0.001 & 0.000 & 0.000 & 0.000 & 0.013 & -0.006 & -0.012 & -0.013 & 0.001 & 0.001 & 0.000 & 0.000 & 0.000 & 0.013 & 0.000 \\
\hline 0.50 & MSE & 0.012 & 0.014 & 0.014 & 0.014 & 0.013 & 0.014 & 0.014 & 0.014 & 0.014 & 0.013 & 0.013 & 0.014 & 0.014 & 0.013 & 0.014 & 0.014 & 0.014 & 0.014 & 0.013 & 0.014 \\
\hline 0.60 & BIAS & 0.009 & -0.012 & 0.000 & -0.012 & -0.002 & 0.000 & 0.000 & 0.000 & 0.000 & 0.010 & -0.007 & -0.012 & -0.012 & -0.002 & 0.000 & 0.000 & 0.000 & 0.000 & 0.010 & 0.000 \\
\hline 0.60 & MSE & 0.010 & 0.011 & 0.011 & 0.011 & 0.010 & 0.011 & 0.011 & 0.011 & 0.011 & 0.010 & 0.010 & 0.011 & 0.011 & 0.010 & 0.011 & 0.011 & 0.011 & 0.011 & 0.010 & 0.011 \\
\hline 0.70 & BIAS & 0.005 & -0.011 & 0.000 & -0.010 & -0.003 & 0.000 & 0.000 & 0.000 & 0.000 & 0.008 & -0.008 & -0.010 & -0.011 & -0.003 & 0.000 & 0.000 & 0.000 & 0.000 & 0.008 & 0.000 \\
\hline 0.70 & MSE & 0.007 & 0.007 & 0.007 & 0.007 & 0.007 & 0.007 & 0.007 & 0.007 & 0.007 & 0.007 & 0.007 & 0.007 & 0.007 & 0.007 & 0.007 & 0.007 & 0.007 & 0.007 & 0.007 & 0.007 \\
\hline 0.80 & BIAS & 0.002 & -0.008 & 0.000 & -0.008 & -0.003 & 0.000 & 0.000 & 0.000 & 0.000 & 0.005 & -0.007 & -0.008 & -0.008 & -0.003 & 0.000 & 0.000 & 0.000 & 0.000 & 0.005 & 0.000 \\
\hline 0.80 & MSE & 0.003 & 0.004 & 0.004 & 0.004 & 0.004 & 0.004 & 0.004 & 0.004 & 0.004 & 0.003 & 0.004 & 0.004 & 0.004 & 0.004 & 0.004 & 0.004 & 0.004 & 0.004 & 0.003 & 0.004 \\
\hline 0.90 & BIAS & 0.001 & -0.005 & 0.000 & -0.005 & -0.002 & 0.000 & 0.000 & 0.000 & 0.000 & 0.003 & -0.004 & -0.005 & -0.005 & -0.002 & 0.000 & 0.000 & 0.000 & 0.000 & 0.003 & 0.000 \\
\hline 0.90 & MSE & 0.001 & 0.001 & 0.001 & 0.001 & 0.001 & 0.001 & 0.001 & 0.001 & 0.001 & 0.001 & 0.001 & 0.001 & 0.001 & 0.001 & 0.001 & 0.001 & 0.001 & 0.001 & 0.001 & 0.001 \\
\hline
\end{tabular}


Table S12: Results for sample size $N=40$ and number of predictors $p=5$.

\begin{tabular}{|c|c|c|c|c|c|c|c|c|c|c|c|c|c|c|c|c|c|c|c|c|c|}
\hline$\rho^{2}$ & What & $R^{2}$ & $\mathrm{E}$ & OPE & $\mathrm{S}$ & W & OP1 & OP2 & OP5 & $\mathrm{P}$ & C & ML & $\mathrm{S}^{+}$ & $\mathrm{E}^{+}$ & $\mathrm{W}^{+}$ & $\mathrm{OP} 1^{+}$ & $\mathrm{OP}^{+}{ }^{+}$ & $\mathrm{OP}^{+}$ & $\mathrm{P}^{+}$ & $\mathrm{C}^{+}$ & $\mathrm{OPE}^{+}$ \\
\hline 0.00 & BIAS & 0.128 & 0.000 & -0.001 & 0.003 & 0.028 & 0.004 & 0.000 & -0.001 & 0.000 & 0.027 & 0.039 & 0.035 & 0.033 & 0.047 & 0.037 & 0.035 & 0.035 & 0.035 & 0.047 & 0.035 \\
\hline 0.00 & MSE & 0.022 & 0.007 & 0.008 & 0.007 & 0.008 & 0.008 & 0.008 & 0.008 & 0.008 & 0.008 & 0.005 & 0.005 & 0.004 & 0.006 & 0.005 & 0.005 & 0.005 & 0.005 & 0.007 & 0.005 \\
\hline 0.01 & BIAS & 0.126 & -0.001 & -0.001 & 0.003 & 0.027 & 0.004 & 0.000 & -0.001 & 0.000 & 0.027 & 0.035 & 0.031 & 0.029 & 0.044 & 0.033 & 0.032 & 0.031 & 0.031 & 0.045 & 0.031 \\
\hline 0.01 & MSE & 0.022 & 0.008 & 0.009 & 0.008 & 0.008 & 0.008 & 0.009 & 0.009 & 0.009 & 0.009 & 0.006 & 0.005 & 0.005 & 0.007 & 0.006 & 0.005 & 0.005 & 0.005 & 0.007 & 0.005 \\
\hline 0.05 & BIAS & 0.119 & -0.003 & -0.001 & 0.001 & 0.024 & 0.004 & 0.000 & -0.001 & 0.000 & 0.025 & 0.025 & 0.019 & 0.016 & 0.034 & 0.022 & 0.020 & 0.020 & 0.020 & 0.036 & 0.020 \\
\hline 0.05 & MSE & 0.022 & 0.011 & 0.012 & 0.010 & 0.011 & 0.011 & 0.011 & 0.012 & 0.011 & 0.011 & 0.008 & 0.007 & 0.007 & 0.009 & 0.008 & 0.008 & 0.008 & 0.008 & 0.010 & 0.008 \\
\hline 0.10 & BIAS & 0.111 & -0.005 & 0.000 & -0.002 & 0.021 & 0.003 & 0.000 & 0.000 & -0.001 & 0.024 & 0.016 & 0.008 & 0.005 & 0.026 & 0.013 & 0.011 & 0.010 & 0.010 & 0.029 & 0.010 \\
\hline 0.10 & MSE & 0.022 & 0.013 & 0.014 & 0.013 & 0.013 & 0.014 & 0.014 & 0.014 & 0.014 & 0.014 & 0.011 & 0.011 & 0.010 & 0.012 & 0.011 & 0.011 & 0.011 & 0.011 & 0.013 & 0.011 \\
\hline 0.20 & BIAS & 0.095 & -0.008 & 0.000 & -0.005 & 0.015 & 0.002 & 0.000 & 0.000 & -0.001 & 0.021 & 0.007 & -0.003 & -0.006 & 0.016 & 0.005 & 0.002 & 0.002 & 0.002 & 0.022 & 0.002 \\
\hline 0.20 & MSE & 0.022 & 0.017 & 0.018 & 0.017 & 0.016 & 0.017 & 0.018 & 0.018 & 0.018 & 0.017 & 0.016 & 0.016 & 0.016 & 0.016 & 0.016 & 0.017 & 0.017 & 0.017 & 0.017 & 0.017 \\
\hline 0.30 & BIAS & 0.080 & -0.011 & 0.000 & -0.008 & 0.010 & 0.002 & 0.000 & 0.000 & -0.001 & 0.018 & 0.002 & -0.008 & -0.010 & 0.010 & 0.002 & 0.000 & 0.000 & 0.000 & 0.018 & 0.000 \\
\hline 0.30 & MSE & 0.020 & 0.018 & 0.019 & 0.018 & 0.017 & 0.018 & 0.019 & 0.019 & 0.019 & 0.018 & 0.017 & 0.018 & 0.018 & 0.017 & 0.018 & 0.018 & 0.018 & 0.018 & 0.018 & 0.018 \\
\hline 0.40 & BIAS & 0.066 & -0.012 & 0.000 & -0.010 & 0.005 & 0.001 & 0.000 & 0.000 & -0.001 & 0.015 & -0.002 & -0.010 & -0.012 & 0.005 & 0.001 & 0.000 & 0.000 & -0.001 & 0.015 & 0.000 \\
\hline 0.40 & MSE & 0.017 & 0.017 & 0.018 & 0.017 & 0.016 & 0.017 & 0.018 & 0.018 & 0.018 & 0.017 & 0.016 & 0.017 & 0.017 & 0.016 & 0.017 & 0.017 & 0.017 & 0.017 & 0.017 & 0.017 \\
\hline 0.50 & BIAS & 0.053 & -0.013 & 0.000 & -0.011 & 0.002 & 0.001 & 0.000 & 0.000 & -0.001 & 0.013 & -0.005 & -0.011 & -0.013 & 0.002 & 0.001 & 0.000 & 0.000 & -0.001 & 0.013 & 0.000 \\
\hline 0.50 & MSE & 0.014 & 0.015 & 0.015 & 0.015 & 0.014 & 0.015 & 0.015 & 0.015 & 0.015 & 0.014 & 0.014 & 0.015 & 0.015 & 0.014 & 0.015 & 0.015 & 0.015 & 0.015 & 0.014 & 0.015 \\
\hline 0.60 & BIAS & 0.040 & -0.012 & 0.000 & -0.011 & -0.001 & 0.000 & 0.000 & 0.000 & -0.001 & 0.010 & -0.007 & -0.011 & -0.012 & -0.001 & 0.000 & 0.000 & 0.000 & -0.001 & 0.010 & 0.000 \\
\hline 0.60 & MSE & 0.010 & 0.012 & 0.011 & 0.012 & 0.011 & 0.011 & 0.011 & 0.011 & 0.011 & 0.011 & 0.011 & 0.012 & 0.012 & 0.011 & 0.011 & 0.011 & 0.011 & 0.011 & 0.011 & 0.011 \\
\hline 0.70 & BIAS & 0.029 & -0.011 & 0.000 & -0.010 & -0.002 & 0.000 & 0.000 & 0.000 & 0.000 & 0.008 & -0.008 & -0.010 & -0.011 & -0.002 & 0.000 & 0.000 & 0.000 & 0.000 & 0.008 & 0.000 \\
\hline 0.70 & MSE & 0.007 & 0.008 & 0.007 & 0.008 & 0.007 & 0.007 & 0.007 & 0.007 & 0.007 & 0.007 & 0.007 & 0.008 & 0.008 & 0.007 & 0.007 & 0.007 & 0.007 & 0.007 & 0.007 & 0.007 \\
\hline 0.80 & BIAS & 0.018 & -0.008 & 0.000 & -0.008 & -0.003 & 0.000 & 0.000 & 0.000 & 0.000 & 0.005 & -0.007 & -0.008 & -0.008 & -0.003 & 0.000 & 0.000 & 0.000 & 0.000 & 0.005 & 0.000 \\
\hline 0.80 & MSE & 0.003 & 0.004 & 0.004 & 0.004 & 0.004 & 0.004 & 0.004 & 0.004 & 0.004 & 0.004 & 0.004 & 0.004 & 0.004 & 0.004 & 0.004 & 0.004 & 0.004 & 0.004 & 0.004 & 0.004 \\
\hline 0.90 & BIAS & 0.009 & -0.005 & 0.000 & -0.004 & -0.002 & 0.000 & 0.000 & 0.000 & 0.000 & 0.003 & -0.004 & -0.004 & -0.005 & -0.002 & 0.000 & 0.000 & 0.000 & 0.000 & 0.003 & 0.000 \\
\hline 0.90 & MSE & 0.001 & 0.001 & 0.001 & 0.001 & 0.001 & 0.001 & 0.001 & 0.001 & 0.001 & 0.001 & 0.001 & 0.001 & 0.001 & 0.001 & 0.001 & 0.001 & 0.001 & 0.001 & 0.001 & 0.001 \\
\hline
\end{tabular}


Table S13: Results for sample size $N=40$ and number of predictors $p=10$.

\begin{tabular}{|c|c|c|c|c|c|c|c|c|c|c|c|c|c|c|c|c|c|c|c|c|c|}
\hline$\rho^{2}$ & What & $R^{2}$ & $\mathrm{E}$ & OPE & $\mathrm{S}$ & $\mathrm{W}$ & OP1 & OP2 & OP5 & $\mathrm{P}$ & $\mathrm{C}$ & ML & $\mathrm{S}^{+}$ & $\mathrm{E}^{+}$ & $\mathrm{w}^{+}$ & $\mathrm{OP} 1^{+}$ & $\mathrm{OP}{ }^{+}$ & $\mathrm{OP}^{+}$ & $\mathrm{P}^{+}$ & $\mathrm{C}^{+}$ & $\mathrm{OPE}^{+}$ \\
\hline 0.00 & BIAS & 0.257 & 0.000 & 0.000 & 0.009 & 0.034 & 0.005 & 0.001 & 0.000 & 0.000 & 0.027 & 0.059 & 0.056 & 0.052 & 0.068 & 0.057 & 0.055 & 0.055 & 0.055 & 0.067 & 0.055 \\
\hline 0.00 & MSE & 0.075 & 0.017 & 0.019 & 0.017 & 0.017 & 0.018 & 0.019 & 0.019 & 0.018 & 0.018 & 0.011 & 0.010 & 0.010 & 0.013 & 0.011 & 0.010 & 0.010 & 0.010 & 0.013 & 0.010 \\
\hline 0.01 & BIAS & 0.254 & 0.000 & 0.001 & 0.009 & 0.033 & 0.005 & 0.001 & 0.001 & 0.000 & 0.027 & 0.055 & 0.052 & 0.048 & 0.064 & 0.053 & 0.051 & 0.051 & 0.050 & 0.063 & 0.051 \\
\hline 0.01 & MSE & 0.074 & 0.017 & 0.019 & 0.017 & 0.017 & 0.019 & 0.019 & 0.019 & 0.019 & 0.019 & 0.012 & 0.011 & 0.010 & 0.013 & 0.011 & 0.011 & 0.011 & 0.011 & 0.013 & 0.011 \\
\hline 0.05 & BIAS & 0.242 & -0.002 & 0.001 & 0.006 & 0.030 & 0.005 & 0.001 & 0.001 & 0.000 & 0.026 & 0.041 & 0.037 & 0.032 & 0.051 & 0.038 & 0.037 & 0.036 & 0.036 & 0.051 & 0.036 \\
\hline 0.05 & MSE & 0.069 & 0.019 & 0.021 & 0.019 & 0.019 & 0.021 & 0.021 & 0.021 & 0.021 & 0.021 & 0.013 & 0.012 & 0.012 & 0.015 & 0.013 & 0.013 & 0.013 & 0.013 & 0.015 & 0.013 \\
\hline 0.10 & BIAS & 0.228 & -0.004 & 0.001 & 0.004 & 0.026 & 0.005 & 0.001 & 0.001 & 0.000 & 0.024 & 0.028 & 0.023 & 0.017 & 0.039 & 0.025 & 0.023 & 0.023 & 0.022 & 0.039 & 0.023 \\
\hline 0.10 & MSE & 0.064 & 0.021 & 0.023 & 0.021 & 0.021 & 0.023 & 0.023 & 0.023 & 0.023 & 0.022 & 0.016 & 0.015 & 0.015 & 0.017 & 0.016 & 0.016 & 0.016 & 0.016 & 0.018 & 0.016 \\
\hline 0.20 & BIAS & 0.200 & -0.007 & 0.001 & 0.000 & 0.020 & 0.004 & 0.001 & 0.001 & 0.000 & 0.022 & 0.012 & 0.006 & 0.000 & 0.023 & 0.010 & 0.008 & 0.008 & 0.007 & 0.026 & 0.008 \\
\hline 0.20 & MSE & 0.053 & 0.023 & 0.025 & 0.023 & 0.022 & 0.024 & 0.025 & 0.025 & 0.025 & 0.024 & 0.020 & 0.020 & 0.020 & 0.020 & 0.021 & 0.021 & 0.021 & 0.021 & 0.022 & 0.021 \\
\hline 0.30 & BIAS & 0.172 & -0.010 & 0.001 & -0.004 & 0.014 & 0.003 & 0.001 & 0.001 & 0.000 & 0.019 & 0.005 & -0.002 & -0.008 & 0.015 & 0.004 & 0.003 & 0.002 & 0.001 & 0.020 & 0.002 \\
\hline 0.30 & MSE & 0.042 & 0.023 & 0.024 & 0.023 & 0.022 & 0.024 & 0.024 & 0.024 & 0.024 & 0.023 & 0.021 & 0.022 & 0.022 & 0.021 & 0.023 & 0.023 & 0.023 & 0.023 & 0.022 & 0.023 \\
\hline 0.40 & BIAS & 0.145 & -0.011 & 0.001 & -0.006 & 0.009 & 0.002 & 0.001 & 0.001 & 0.000 & 0.016 & 0.000 & -0.006 & -0.011 & 0.009 & 0.002 & 0.001 & 0.001 & 0.000 & 0.016 & 0.001 \\
\hline 0.40 & MSE & 0.033 & 0.021 & 0.021 & 0.021 & 0.020 & 0.021 & 0.021 & 0.021 & 0.021 & 0.021 & 0.020 & 0.020 & 0.021 & 0.019 & 0.021 & 0.021 & 0.021 & 0.021 & 0.020 & 0.021 \\
\hline 0.50 & BIAS & 0.119 & -0.012 & 0.001 & -0.008 & 0.005 & 0.001 & 0.001 & 0.001 & 0.000 & 0.013 & -0.003 & -0.008 & -0.012 & 0.005 & 0.001 & 0.001 & 0.001 & 0.000 & 0.013 & 0.001 \\
\hline 0.50 & MSE & 0.024 & 0.018 & 0.018 & 0.017 & 0.016 & 0.017 & 0.018 & 0.018 & 0.018 & 0.017 & 0.017 & 0.017 & 0.018 & 0.016 & 0.017 & 0.018 & 0.018 & 0.018 & 0.017 & 0.018 \\
\hline 0.60 & BIAS & 0.094 & -0.012 & 0.000 & -0.008 & 0.002 & 0.001 & 0.001 & 0.000 & 0.000 & 0.011 & -0.005 & -0.008 & -0.012 & 0.002 & 0.001 & 0.001 & 0.000 & 0.000 & 0.011 & 0.000 \\
\hline 0.60 & MSE & 0.016 & 0.013 & 0.013 & 0.013 & 0.012 & 0.013 & 0.013 & 0.013 & 0.013 & 0.013 & 0.013 & 0.013 & 0.013 & 0.012 & 0.013 & 0.013 & 0.013 & 0.013 & 0.013 & 0.013 \\
\hline 0.70 & BIAS & 0.069 & -0.011 & 0.000 & -0.008 & 0.000 & 0.001 & 0.000 & 0.000 & 0.000 & 0.008 & -0.006 & -0.008 & -0.011 & 0.000 & 0.001 & 0.000 & 0.000 & 0.000 & 0.008 & 0.000 \\
\hline 0.70 & MSE & 0.010 & 0.009 & 0.008 & 0.009 & 0.008 & 0.008 & 0.008 & 0.008 & 0.008 & 0.008 & 0.008 & 0.009 & 0.009 & 0.008 & 0.008 & 0.008 & 0.008 & 0.008 & 0.008 & 0.008 \\
\hline 0.80 & BIAS & 0.045 & -0.008 & 0.000 & -0.006 & -0.001 & 0.000 & 0.000 & 0.000 & 0.000 & 0.005 & -0.005 & -0.006 & -0.008 & -0.001 & 0.000 & 0.000 & 0.000 & 0.000 & 0.005 & 0.000 \\
\hline 0.80 & MSE & 0.005 & 0.005 & 0.004 & 0.004 & 0.004 & 0.004 & 0.004 & 0.004 & 0.004 & 0.004 & 0.004 & 0.004 & 0.005 & 0.004 & 0.004 & 0.004 & 0.004 & 0.004 & 0.004 & 0.004 \\
\hline 0.90 & BIAS & 0.022 & -0.005 & 0.000 & -0.004 & -0.001 & 0.000 & 0.000 & 0.000 & 0.000 & 0.003 & -0.004 & -0.004 & -0.005 & -0.001 & 0.000 & 0.000 & 0.000 & 0.000 & 0.003 & 0.000 \\
\hline 0.90 & MSE & 0.001 & 0.001 & 0.001 & 0.001 & 0.001 & 0.001 & 0.001 & 0.001 & 0.001 & 0.001 & 0.001 & 0.001 & 0.001 & 0.001 & 0.001 & 0.001 & 0.001 & 0.001 & 0.001 & 0.001 \\
\hline
\end{tabular}


Table S14: Results for sample size $N=50$ and number of predictors $p=2$.

\begin{tabular}{|c|c|c|c|c|c|c|c|c|c|c|c|c|c|c|c|c|c|c|c|c|c|}
\hline$\rho^{2}$ & What & $R^{2}$ & $\mathrm{E}$ & OPE & $\mathrm{S}$ & $\mathrm{W}$ & OP1 & OP2 & OP5 & $\mathrm{P}$ & C & ML & $\mathrm{S}^{+}$ & $\mathrm{E}^{+}$ & $\mathrm{W}^{+}$ & $\mathrm{OP} 1^{+}$ & $\mathrm{OP} 2^{+}$ & $\mathrm{OP}^{+}$ & $\mathrm{P}^{+}$ & $\mathrm{C}^{+}$ & $\mathrm{OPE}^{+}$ \\
\hline 0.00 & BIAS & 0.041 & 0.000 & 0.000 & 0.001 & 0.021 & 0.003 & 0.000 & 0.000 & 0.000 & 0.022 & 0.020 & 0.016 & 0.015 & 0.025 & 0.017 & 0.016 & 0.016 & 0.016 & 0.026 & 0.016 \\
\hline 0.00 & MSE & 0.003 & 0.002 & 0.002 & 0.002 & 0.002 & 0.002 & 0.002 & 0.002 & 0.002 & 0.002 & 0.002 & 0.001 & 0.001 & 0.002 & 0.001 & 0.001 & 0.001 & 0.001 & 0.002 & 0.001 \\
\hline 0.01 & BIAS & 0.040 & 0.000 & 0.000 & 0.000 & 0.020 & 0.003 & 0.000 & 0.000 & 0.000 & 0.021 & 0.018 & 0.013 & 0.012 & 0.024 & 0.015 & 0.014 & 0.013 & 0.014 & 0.025 & 0.013 \\
\hline 0.01 & MSE & 0.004 & 0.002 & 0.003 & 0.002 & 0.003 & 0.003 & 0.003 & 0.003 & 0.003 & 0.003 & 0.002 & 0.002 & 0.002 & 0.003 & 0.002 & 0.002 & 0.002 & 0.002 & 0.003 & 0.002 \\
\hline 0.05 & BIAS & 0.037 & -0.002 & 0.000 & -0.001 & 0.018 & 0.003 & 0.000 & 0.000 & 0.000 & 0.021 & 0.014 & 0.005 & 0.004 & 0.019 & 0.008 & 0.007 & 0.006 & 0.007 & 0.022 & 0.006 \\
\hline 0.05 & MSE & 0.006 & 0.005 & 0.005 & 0.005 & 0.005 & 0.005 & 0.005 & 0.005 & 0.005 & 0.005 & 0.005 & 0.004 & 0.004 & 0.005 & 0.004 & 0.004 & 0.004 & 0.004 & 0.005 & 0.004 \\
\hline 0.10 & BIAS & 0.033 & -0.003 & 0.000 & -0.003 & 0.015 & 0.003 & 0.000 & 0.000 & 0.000 & 0.019 & 0.011 & -0.001 & -0.001 & 0.016 & 0.004 & 0.003 & 0.002 & 0.003 & 0.020 & 0.002 \\
\hline 0.10 & MSE & 0.008 & 0.007 & 0.008 & 0.007 & 0.007 & 0.008 & 0.008 & 0.008 & 0.008 & 0.008 & 0.007 & 0.007 & 0.007 & 0.007 & 0.007 & 0.007 & 0.007 & 0.007 & 0.008 & 0.007 \\
\hline 0.20 & BIAS & 0.027 & -0.006 & 0.000 & -0.005 & 0.011 & 0.002 & 0.000 & 0.000 & 0.000 & 0.017 & 0.006 & -0.005 & -0.006 & 0.011 & 0.002 & 0.001 & 0.001 & 0.000 & 0.017 & 0.001 \\
\hline 0.20 & MSE & 0.011 & 0.011 & 0.011 & 0.011 & 0.011 & 0.011 & 0.011 & 0.011 & 0.011 & 0.011 & 0.010 & 0.011 & 0.011 & 0.010 & 0.011 & 0.011 & 0.011 & 0.011 & 0.011 & 0.011 \\
\hline 0.30 & BIAS & 0.021 & -0.008 & 0.000 & -0.007 & 0.007 & 0.002 & 0.000 & 0.000 & 0.000 & 0.015 & 0.002 & -0.007 & -0.008 & 0.007 & 0.002 & 0.000 & 0.000 & 0.000 & 0.015 & 0.000 \\
\hline 0.30 & MSE & 0.012 & 0.012 & 0.013 & 0.012 & 0.012 & 0.013 & 0.013 & 0.013 & 0.013 & 0.012 & 0.012 & 0.012 & 0.012 & 0.012 & 0.013 & 0.013 & 0.013 & 0.013 & 0.012 & 0.013 \\
\hline 0.40 & BIAS & 0.016 & -0.009 & 0.000 & -0.009 & 0.003 & 0.001 & 0.000 & 0.000 & 0.000 & 0.013 & -0.001 & -0.009 & -0.009 & 0.003 & 0.001 & 0.000 & 0.000 & 0.000 & 0.013 & 0.000 \\
\hline 0.40 & MSE & 0.011 & 0.012 & 0.012 & 0.012 & 0.012 & 0.012 & 0.012 & 0.012 & 0.012 & 0.012 & 0.012 & 0.012 & 0.012 & 0.012 & 0.012 & 0.012 & 0.012 & 0.012 & 0.012 & 0.012 \\
\hline 0.50 & BIAS & 0.011 & -0.010 & 0.000 & -0.009 & 0.001 & 0.001 & 0.000 & 0.000 & 0.000 & 0.011 & -0.004 & -0.009 & -0.010 & 0.001 & 0.001 & 0.000 & 0.000 & 0.000 & 0.011 & 0.000 \\
\hline 0.50 & MSE & 0.010 & 0.011 & 0.011 & 0.011 & 0.010 & 0.011 & 0.011 & 0.011 & 0.011 & 0.010 & 0.010 & 0.011 & 0.011 & 0.010 & 0.011 & 0.011 & 0.011 & 0.011 & 0.010 & 0.011 \\
\hline 0.60 & BIAS & 0.007 & -0.010 & 0.000 & -0.009 & -0.001 & 0.001 & 0.000 & 0.000 & 0.000 & 0.009 & -0.006 & -0.009 & -0.010 & -0.001 & 0.001 & 0.000 & 0.000 & 0.000 & 0.009 & 0.000 \\
\hline 0.60 & MSE & 0.008 & 0.008 & 0.008 & 0.008 & 0.008 & 0.008 & 0.008 & 0.008 & 0.008 & 0.008 & 0.008 & 0.008 & 0.008 & 0.008 & 0.008 & 0.008 & 0.008 & 0.008 & 0.008 & 0.008 \\
\hline 0.70 & BIAS & 0.004 & -0.008 & 0.000 & -0.008 & -0.002 & 0.000 & 0.000 & 0.000 & 0.000 & 0.007 & -0.006 & -0.008 & -0.008 & -0.002 & 0.000 & 0.000 & 0.000 & 0.000 & 0.007 & 0.000 \\
\hline 0.70 & MSE & 0.005 & 0.006 & 0.005 & 0.006 & 0.005 & 0.005 & 0.005 & 0.005 & 0.005 & 0.005 & 0.006 & 0.006 & 0.006 & 0.005 & 0.005 & 0.005 & 0.005 & 0.005 & 0.005 & 0.005 \\
\hline 0.80 & BIAS & 0.002 & -0.006 & 0.000 & -0.006 & -0.002 & 0.000 & 0.000 & 0.000 & 0.000 & 0.004 & -0.005 & -0.006 & -0.006 & -0.002 & 0.000 & 0.000 & 0.000 & 0.000 & 0.004 & 0.000 \\
\hline 0.80 & MSE & 0.003 & 0.003 & 0.003 & 0.003 & 0.003 & 0.003 & 0.003 & 0.003 & 0.003 & 0.003 & 0.003 & 0.003 & 0.003 & 0.003 & 0.003 & 0.003 & 0.003 & 0.003 & 0.003 & 0.003 \\
\hline 0.90 & BIAS & & -0.004 & & -0.004 & -0.002 & & & & 0.000 & & & & & -0.002 & & 0.000 & & & 0.002 & 0.000 \\
\hline 0.90 & MSE & 0.001 & 0.001 & 0.001 & 0.001 & 0.001 & 0.001 & 0.001 & 0.001 & 0.001 & 0.001 & 0.001 & 0.001 & 0.001 & 0.001 & 0.001 & 0.001 & 0.001 & 0.001 & 0.001 & 0.001 \\
\hline
\end{tabular}


Table S15: Results for sample size $N=50$ and number of predictors $p=5$.

\begin{tabular}{|c|c|c|c|c|c|c|c|c|c|c|c|c|c|c|c|c|c|c|c|c|c|}
\hline$\rho^{2}$ & What & $R^{2}$ & $\mathrm{E}$ & OPE & $\mathrm{S}$ & $\mathrm{W}$ & OP1 & OP2 & OP5 & $\mathrm{P}$ & C & ML & $\mathrm{S}^{+}$ & $\mathrm{E}^{+}$ & $\mathrm{W}^{+}$ & $\mathrm{OP} 1^{+}$ & $\mathrm{OP} 2^{+}$ & $\mathrm{OP}^{+}$ & $\mathrm{P}^{+}$ & $\mathrm{C}^{+}$ & $\mathrm{OPE}^{+}$ \\
\hline 0.00 & BIAS & 0.102 & 0.000 & 0.000 & 0.002 & 0.022 & 0.003 & 0.000 & 0.000 & 0.000 & 0.021 & 0.030 & 0.027 & 0.026 & 0.036 & 0.028 & 0.027 & 0.027 & 0.027 & 0.037 & 0.027 \\
\hline 0.00 & MSE & 0.014 & 0.004 & 0.005 & 0.004 & 0.005 & 0.005 & 0.005 & 0.005 & 0.005 & 0.005 & 0.003 & 0.003 & 0.003 & 0.004 & 0.003 & 0.003 & 0.003 & 0.003 & 0.004 & 0.003 \\
\hline 0.01 & BIAS & 0.100 & -0.001 & 0.000 & 0.002 & 0.021 & 0.003 & 0.000 & 0.000 & 0.000 & 0.021 & 0.027 & 0.023 & 0.022 & 0.034 & 0.025 & 0.024 & 0.024 & 0.024 & 0.035 & 0.024 \\
\hline 0.01 & MSE & 0.014 & 0.005 & 0.005 & 0.005 & 0.005 & 0.005 & 0.005 & 0.005 & 0.005 & 0.006 & 0.004 & 0.003 & 0.003 & 0.004 & 0.004 & 0.003 & 0.003 & 0.003 & 0.005 & 0.003 \\
\hline 0.05 & BIAS & 0.095 & -0.002 & 0.000 & 0.000 & 0.019 & 0.002 & 0.000 & 0.000 & 0.000 & 0.020 & 0.018 & 0.012 & 0.011 & 0.026 & 0.015 & 0.013 & 0.013 & 0.013 & 0.027 & 0.013 \\
\hline 0.05 & MSE & 0.015 & 0.007 & 0.008 & 0.007 & 0.007 & 0.008 & 0.008 & 0.008 & 0.008 & 0.008 & 0.006 & 0.005 & 0.005 & 0.006 & 0.006 & 0.006 & 0.006 & 0.006 & 0.007 & 0.006 \\
\hline 0.10 & BIAS & 0.088 & -0.004 & 0.000 & -0.002 & 0.016 & 0.002 & 0.000 & 0.000 & 0.000 & 0.019 & 0.011 & 0.004 & 0.002 & 0.019 & 0.008 & 0.006 & 0.006 & 0.006 & 0.022 & 0.006 \\
\hline 0.10 & MSE & 0.016 & 0.010 & 0.010 & 0.010 & 0.010 & 0.010 & 0.010 & 0.010 & 0.010 & 0.010 & 0.009 & 0.008 & 0.008 & 0.009 & 0.009 & 0.009 & 0.009 & 0.009 & 0.009 & 0.009 \\
\hline 0.20 & BIAS & 0.076 & -0.007 & 0.000 & -0.005 & 0.011 & 0.001 & 0.000 & 0.000 & 0.000 & 0.017 & 0.005 & -0.004 & -0.006 & 0.012 & 0.002 & 0.001 & 0.001 & 0.001 & 0.017 & 0.001 \\
\hline 0.20 & MSE & 0.016 & 0.013 & 0.013 & 0.013 & 0.012 & 0.013 & 0.013 & 0.013 & 0.013 & 0.013 & 0.012 & 0.012 & 0.012 & 0.012 & 0.013 & 0.013 & 0.013 & 0.013 & 0.013 & 0.013 \\
\hline 0.30 & BIAS & 0.064 & -0.009 & 0.000 & -0.007 & 0.007 & 0.001 & 0.000 & 0.000 & -0.001 & 0.014 & 0.001 & -0.007 & -0.009 & 0.007 & 0.001 & 0.000 & 0.000 & 0.000 & 0.014 & 0.000 \\
\hline 0.30 & MSE & 0.015 & 0.014 & 0.014 & 0.014 & 0.013 & 0.014 & 0.014 & 0.014 & 0.014 & 0.014 & 0.013 & 0.014 & 0.014 & 0.013 & 0.014 & 0.014 & 0.014 & 0.014 & 0.014 & 0.014 \\
\hline 0.40 & BIAS & 0.052 & -0.010 & 0.000 & -0.009 & 0.004 & 0.000 & 0.000 & 0.000 & -0.001 & 0.012 & -0.002 & -0.009 & -0.010 & 0.004 & 0.000 & 0.000 & 0.000 & -0.001 & 0.012 & 0.000 \\
\hline 0.40 & MSE & 0.013 & 0.013 & 0.014 & 0.013 & 0.013 & 0.013 & 0.014 & 0.014 & 0.014 & 0.013 & 0.013 & 0.013 & 0.013 & 0.013 & 0.013 & 0.013 & 0.014 & 0.014 & 0.013 & 0.014 \\
\hline 0.50 & BIAS & 0.042 & -0.010 & 0.000 & -0.009 & 0.001 & 0.000 & 0.000 & 0.000 & -0.001 & 0.010 & -0.004 & -0.009 & -0.010 & 0.001 & 0.000 & 0.000 & 0.000 & -0.001 & 0.010 & 0.000 \\
\hline 0.50 & MSE & 0.011 & 0.012 & 0.012 & 0.012 & 0.011 & 0.011 & 0.012 & 0.012 & 0.012 & 0.011 & 0.011 & 0.012 & 0.012 & 0.011 & 0.011 & 0.012 & 0.012 & 0.012 & 0.011 & 0.012 \\
\hline 0.60 & BIAS & 0.032 & -0.010 & 0.000 & -0.009 & -0.001 & 0.000 & 0.000 & 0.000 & -0.001 & 0.008 & -0.006 & -0.009 & -0.010 & -0.001 & 0.000 & 0.000 & 0.000 & -0.001 & 0.008 & 0.000 \\
\hline 0.60 & MSE & 0.008 & 0.009 & 0.009 & 0.009 & 0.009 & 0.009 & 0.009 & 0.009 & 0.009 & 0.008 & 0.009 & 0.009 & 0.009 & 0.009 & 0.009 & 0.009 & 0.009 & 0.009 & 0.008 & 0.009 \\
\hline 0.70 & BIAS & 0.023 & -0.009 & 0.000 & -0.008 & -0.002 & 0.000 & 0.000 & 0.000 & 0.000 & 0.006 & -0.006 & -0.008 & -0.009 & -0.002 & 0.000 & 0.000 & 0.000 & 0.000 & 0.006 & 0.000 \\
\hline 0.70 & MSE & 0.005 & 0.006 & 0.006 & 0.006 & 0.006 & 0.006 & 0.006 & 0.006 & 0.006 & 0.006 & 0.006 & 0.006 & 0.006 & 0.006 & 0.006 & 0.006 & 0.006 & 0.006 & 0.006 & 0.006 \\
\hline 0.80 & BIAS & 0.014 & -0.007 & 0.000 & -0.006 & -0.002 & 0.000 & 0.000 & 0.000 & 0.000 & 0.004 & -0.005 & -0.006 & -0.007 & -0.002 & 0.000 & 0.000 & 0.000 & 0.000 & 0.004 & 0.000 \\
\hline 0.80 & MSE & 0.003 & 0.003 & 0.003 & 0.003 & 0.003 & 0.003 & 0.003 & 0.003 & 0.003 & 0.003 & 0.003 & 0.003 & 0.003 & 0.003 & 0.003 & 0.003 & 0.003 & 0.003 & 0.003 & 0.003 \\
\hline 0.90 & BIAS & 0.007 & -0.004 & 0.000 & -0.004 & -0.002 & 0.000 & 0.000 & 0.000 & 0.000 & 0.002 & -0.003 & -0.004 & -0.004 & -0.002 & 0.000 & 0.000 & 0.000 & 0.000 & 0.002 & 0.000 \\
\hline 0.90 & MSE & 0.001 & 0.001 & 0.001 & 0.001 & 0.001 & 0.001 & 0.001 & 0.001 & 0.001 & 0.001 & 0.001 & 0.001 & 0.001 & 0.001 & 0.001 & 0.001 & 0.001 & 0.001 & 0.001 & 0.001 \\
\hline
\end{tabular}


Table S16: Results for sample size $N=50$ and number of predictors $p=10$.

\begin{tabular}{|c|c|c|c|c|c|c|c|c|c|c|c|c|c|c|c|c|c|c|c|c|c|}
\hline$\rho^{2}$ & What & $R^{2}$ & $\mathrm{E}$ & OPE & $\mathrm{S}$ & $\mathrm{W}$ & OP1 & OP2 & OP5 & $\mathrm{P}$ & C & ML & $\mathrm{S}^{+}$ & $\mathrm{E}^{+}$ & $\mathrm{W}^{+}$ & $\mathrm{OP} 1^{+}$ & $\mathrm{OP} 2^{+}$ & $\mathrm{OP}^{+}$ & $\mathrm{P}^{+}$ & $\mathrm{C}^{+}$ & $\mathrm{OPE}^{+}$ \\
\hline 0.00 & BIAS & 0.204 & 0.001 & 0.001 & 0.006 & 0.025 & 0.004 & 0.001 & 0.001 & 0.000 & 0.022 & 0.045 & 0.043 & 0.040 & 0.052 & 0.043 & 0.042 & 0.042 & 0.042 & 0.052 & 0.042 \\
\hline 0.00 & MSE & 0.048 & 0.010 & 0.011 & 0.010 & 0.010 & 0.011 & 0.011 & 0.011 & 0.011 & 0.011 & 0.007 & 0.006 & 0.006 & 0.008 & 0.006 & 0.006 & 0.006 & 0.006 & 0.008 & 0.006 \\
\hline 0.01 & BIAS & 0.202 & 0.000 & 0.001 & 0.005 & 0.025 & 0.004 & 0.001 & 0.001 & 0.001 & 0.022 & 0.042 & 0.039 & 0.036 & 0.049 & 0.039 & 0.038 & 0.038 & 0.038 & 0.048 & 0.038 \\
\hline 0.01 & MSE & 0.048 & 0.011 & 0.012 & 0.011 & 0.011 & 0.011 & 0.011 & 0.012 & 0.011 & 0.011 & 0.007 & 0.007 & 0.006 & 0.008 & 0.007 & 0.007 & 0.007 & 0.007 & 0.008 & 0.007 \\
\hline 0.05 & BIAS & 0.193 & -0.001 & 0.001 & 0.004 & 0.023 & 0.004 & 0.001 & 0.001 & 0.001 & 0.021 & 0.029 & 0.025 & 0.022 & 0.037 & 0.026 & 0.025 & 0.025 & 0.025 & 0.037 & 0.025 \\
\hline 0.05 & MSE & 0.045 & 0.013 & 0.013 & 0.012 & 0.012 & 0.013 & 0.013 & 0.013 & 0.013 & 0.013 & 0.009 & 0.008 & 0.008 & 0.010 & 0.009 & 0.009 & 0.009 & 0.009 & 0.010 & 0.009 \\
\hline 0.10 & BIAS & 0.181 & -0.003 & 0.001 & 0.002 & 0.020 & 0.003 & 0.001 & 0.001 & 0.000 & 0.020 & 0.018 & 0.013 & 0.010 & 0.027 & 0.015 & 0.014 & 0.014 & 0.014 & 0.028 & 0.014 \\
\hline 0.10 & MSE & 0.042 & 0.014 & 0.015 & 0.014 & 0.014 & 0.015 & 0.015 & 0.015 & 0.015 & 0.015 & 0.012 & 0.011 & 0.011 & 0.012 & 0.012 & 0.012 & 0.012 & 0.011 & 0.013 & 0.012 \\
\hline 0.20 & BIAS & 0.159 & -0.006 & 0.001 & -0.001 & 0.015 & 0.003 & 0.001 & 0.001 & 0.000 & 0.017 & 0.008 & 0.001 & -0.003 & 0.016 & 0.005 & 0.004 & 0.004 & 0.003 & 0.019 & 0.004 \\
\hline 0.20 & MSE & 0.036 & 0.017 & 0.017 & 0.016 & 0.016 & 0.017 & 0.017 & 0.017 & 0.017 & 0.017 & 0.015 & 0.015 & 0.015 & 0.015 & 0.016 & 0.016 & 0.016 & 0.016 & 0.016 & 0.016 \\
\hline 0.30 & BIAS & 0.137 & -0.008 & 0.001 & -0.004 & 0.010 & 0.002 & 0.001 & 0.001 & 0.000 & 0.015 & 0.003 & -0.004 & -0.007 & 0.010 & 0.002 & 0.001 & 0.001 & 0.001 & 0.015 & 0.001 \\
\hline 0.30 & MSE & 0.029 & 0.017 & 0.017 & 0.017 & 0.016 & 0.017 & 0.017 & 0.017 & 0.017 & 0.017 & 0.016 & 0.016 & 0.017 & 0.016 & 0.017 & 0.017 & 0.017 & 0.017 & 0.017 & 0.017 \\
\hline 0.40 & BIAS & 0.115 & -0.009 & 0.001 & -0.006 & 0.006 & 0.001 & 0.001 & 0.001 & 0.000 & 0.013 & 0.000 & -0.006 & -0.009 & 0.006 & 0.001 & 0.001 & 0.001 & 0.000 & 0.013 & 0.001 \\
\hline 0.40 & MSE & 0.023 & 0.016 & 0.016 & 0.015 & 0.015 & 0.016 & 0.016 & 0.016 & 0.016 & 0.015 & 0.015 & 0.015 & 0.016 & 0.015 & 0.016 & 0.016 & 0.016 & 0.016 & 0.015 & 0.016 \\
\hline 0.50 & BIAS & 0.094 & -0.010 & 0.001 & -0.007 & 0.003 & 0.001 & 0.001 & 0.001 & 0.000 & 0.011 & -0.003 & -0.007 & -0.010 & 0.003 & 0.001 & 0.001 & 0.001 & 0.000 & 0.011 & 0.001 \\
\hline 0.50 & MSE & 0.017 & 0.013 & 0.013 & 0.013 & 0.012 & 0.013 & 0.013 & 0.013 & 0.013 & 0.013 & 0.013 & 0.013 & 0.013 & 0.012 & 0.013 & 0.013 & 0.013 & 0.013 & 0.013 & 0.013 \\
\hline 0.60 & BIAS & 0.074 & -0.009 & 0.000 & -0.007 & 0.001 & 0.001 & 0.000 & 0.000 & 0.000 & 0.009 & -0.004 & -0.007 & -0.009 & 0.001 & 0.001 & 0.000 & 0.000 & 0.000 & 0.009 & 0.000 \\
\hline 0.60 & MSE & 0.012 & 0.010 & 0.010 & 0.010 & 0.009 & 0.010 & 0.010 & 0.010 & 0.010 & 0.009 & 0.010 & 0.010 & 0.010 & 0.009 & 0.010 & 0.010 & 0.010 & 0.010 & 0.009 & 0.010 \\
\hline 0.70 & BIAS & 0.055 & -0.008 & 0.000 & -0.007 & -0.001 & 0.000 & 0.000 & 0.000 & 0.000 & 0.006 & -0.005 & -0.007 & -0.008 & -0.001 & 0.000 & 0.000 & 0.000 & 0.000 & 0.006 & 0.000 \\
\hline 0.70 & MSE & 0.007 & 0.007 & 0.006 & 0.006 & 0.006 & 0.006 & 0.006 & 0.006 & 0.006 & 0.006 & 0.006 & 0.006 & 0.007 & 0.006 & 0.006 & 0.006 & 0.006 & 0.006 & 0.006 & 0.006 \\
\hline 0.80 & BIAS & 0.036 & -0.006 & 0.000 & -0.005 & -0.001 & 0.000 & 0.000 & 0.000 & 0.000 & 0.004 & -0.005 & -0.005 & -0.006 & -0.001 & 0.000 & 0.000 & 0.000 & 0.000 & 0.004 & 0.000 \\
\hline 0.80 & MSE & 0.003 & 0.003 & 0.003 & 0.003 & 0.003 & 0.003 & 0.003 & 0.003 & 0.003 & 0.003 & 0.003 & 0.003 & 0.003 & 0.003 & 0.003 & 0.003 & 0.003 & 0.003 & 0.003 & 0.003 \\
\hline 0.90 & BIAS & 0.017 & -0.004 & & -0.003 & -0.001 & & 0.000 & & 0.000 & & -0.003 & & & -0.001 & & 0.000 & & & 0.002 & 0.000 \\
\hline 0.90 & MSE & 0.001 & 0.001 & 0.001 & 0.001 & 0.001 & 0.001 & 0.001 & 0.001 & 0.001 & 0.001 & 0.001 & 0.001 & 0.001 & 0.001 & 0.001 & 0.001 & 0.001 & 0.001 & 0.001 & 0.001 \\
\hline
\end{tabular}


Table S17: Results for sample size $N=60$ and number of predictors $p=2$.

\begin{tabular}{|c|c|c|c|c|c|c|c|c|c|c|c|c|c|c|c|c|c|c|c|c|c|}
\hline$\rho^{2}$ & What & $R^{2}$ & $\mathrm{E}$ & OPE & $\mathrm{S}$ & $\mathrm{W}$ & OP1 & OP2 & OP5 & $\mathrm{P}$ & C & ML & $\mathrm{S}^{+}$ & $\mathrm{E}^{+}$ & $\mathrm{W}^{+}$ & $\mathrm{OP} 1^{+}$ & $\mathrm{OP} 2^{+}$ & $\mathrm{OP}^{+}$ & $\mathrm{P}^{+}$ & $\mathrm{C}^{+}$ & $\mathrm{OPE}^{+}$ \\
\hline 0.00 & BIAS & 0.034 & 0.000 & 0.000 & 0.001 & 0.017 & 0.002 & 0.000 & 0.000 & 0.000 & 0.018 & 0.016 & 0.013 & 0.013 & 0.021 & 0.014 & 0.013 & 0.013 & 0.013 & 0.022 & 0.013 \\
\hline 0.00 & MSE & 0.002 & 0.001 & 0.001 & 0.001 & 0.001 & 0.001 & 0.001 & 0.001 & 0.001 & 0.002 & 0.001 & 0.001 & 0.001 & 0.001 & 0.001 & 0.001 & 0.001 & 0.001 & 0.001 & 0.001 \\
\hline 0.01 & BIAS & 0.033 & 0.000 & 0.000 & 0.000 & 0.017 & 0.002 & 0.000 & 0.000 & 0.000 & 0.018 & 0.015 & 0.010 & 0.010 & 0.020 & 0.012 & 0.011 & 0.011 & 0.011 & 0.021 & 0.011 \\
\hline 0.01 & MSE & 0.003 & 0.002 & 0.002 & 0.002 & 0.002 & 0.002 & 0.002 & 0.002 & 0.002 & 0.002 & 0.002 & 0.001 & 0.001 & 0.002 & 0.001 & 0.001 & 0.001 & 0.001 & 0.002 & 0.001 \\
\hline 0.05 & BIAS & 0.031 & -0.002 & 0.000 & -0.001 & 0.015 & 0.002 & 0.000 & 0.000 & 0.000 & 0.017 & 0.011 & 0.003 & 0.003 & 0.016 & 0.006 & 0.004 & 0.004 & 0.004 & 0.018 & 0.004 \\
\hline 0.05 & MSE & 0.005 & 0.004 & 0.004 & 0.004 & 0.004 & 0.004 & 0.004 & 0.004 & 0.004 & 0.004 & 0.004 & 0.003 & 0.003 & 0.004 & 0.004 & 0.004 & 0.004 & 0.004 & 0.004 & 0.004 \\
\hline 0.10 & BIAS & 0.028 & -0.003 & 0.000 & -0.002 & 0.013 & 0.002 & 0.000 & 0.000 & 0.000 & 0.016 & 0.009 & -0.001 & -0.002 & 0.013 & 0.003 & 0.001 & 0.001 & 0.001 & 0.016 & 0.001 \\
\hline 0.10 & MSE & 0.006 & 0.006 & 0.006 & 0.006 & 0.006 & 0.006 & 0.006 & 0.006 & 0.006 & 0.006 & 0.006 & 0.006 & 0.006 & 0.006 & 0.006 & 0.006 & 0.006 & 0.006 & 0.006 & 0.006 \\
\hline 0.20 & BIAS & 0.022 & -0.005 & 0.000 & -0.005 & 0.009 & 0.001 & 0.000 & 0.000 & 0.000 & 0.014 & 0.005 & -0.005 & -0.005 & 0.009 & 0.001 & 0.000 & 0.000 & 0.000 & 0.014 & 0.000 \\
\hline 0.20 & MSE & 0.009 & 0.009 & 0.009 & 0.009 & 0.009 & 0.009 & 0.009 & 0.009 & 0.009 & 0.009 & 0.009 & 0.009 & 0.009 & 0.009 & 0.009 & 0.009 & 0.009 & 0.009 & 0.009 & 0.009 \\
\hline 0.30 & BIAS & 0.017 & -0.007 & 0.000 & -0.007 & 0.005 & 0.001 & 0.000 & 0.000 & 0.000 & 0.012 & 0.002 & -0.007 & -0.007 & 0.005 & 0.001 & 0.000 & 0.000 & 0.000 & 0.012 & 0.000 \\
\hline 0.30 & MSE & 0.010 & 0.010 & 0.010 & 0.010 & 0.010 & 0.010 & 0.010 & 0.010 & 0.010 & 0.010 & 0.010 & 0.010 & 0.010 & 0.010 & 0.010 & 0.010 & 0.010 & 0.010 & 0.010 & 0.010 \\
\hline 0.40 & BIAS & 0.013 & -0.008 & 0.000 & -0.008 & 0.002 & 0.001 & 0.000 & 0.000 & 0.000 & 0.010 & -0.002 & -0.008 & -0.008 & 0.002 & 0.001 & 0.000 & 0.000 & 0.000 & 0.010 & 0.000 \\
\hline 0.40 & MSE & 0.010 & 0.010 & 0.010 & 0.010 & 0.010 & 0.010 & 0.010 & 0.010 & 0.010 & 0.010 & 0.010 & 0.010 & 0.010 & 0.010 & 0.010 & 0.010 & 0.010 & 0.010 & 0.010 & 0.010 \\
\hline 0.50 & BIAS & 0.009 & -0.008 & 0.000 & -0.008 & 0.000 & 0.000 & 0.000 & 0.000 & 0.000 & 0.009 & -0.004 & -0.008 & -0.008 & 0.000 & 0.000 & 0.000 & 0.000 & 0.000 & 0.009 & 0.000 \\
\hline 0.50 & MSE & 0.008 & 0.009 & 0.009 & 0.009 & 0.009 & 0.009 & 0.009 & 0.009 & 0.009 & 0.009 & 0.009 & 0.009 & 0.009 & 0.009 & 0.009 & 0.009 & 0.009 & 0.009 & 0.009 & 0.009 \\
\hline 0.60 & BIAS & 0.006 & -0.008 & 0.000 & -0.008 & -0.001 & 0.000 & 0.000 & 0.000 & 0.000 & 0.007 & -0.005 & -0.008 & -0.008 & -0.001 & 0.000 & 0.000 & 0.000 & 0.000 & 0.007 & 0.000 \\
\hline 0.60 & MSE & 0.006 & 0.007 & 0.007 & 0.007 & 0.007 & 0.007 & 0.007 & 0.007 & 0.007 & 0.007 & 0.007 & 0.007 & 0.007 & 0.007 & 0.007 & 0.007 & 0.007 & 0.007 & 0.007 & 0.007 \\
\hline 0.70 & BIAS & 0.003 & -0.007 & 0.000 & -0.007 & -0.002 & 0.000 & 0.000 & 0.000 & 0.000 & 0.005 & -0.005 & -0.007 & -0.007 & -0.002 & 0.000 & 0.000 & 0.000 & 0.000 & 0.005 & 0.000 \\
\hline 0.70 & MSE & 0.004 & 0.005 & 0.004 & 0.005 & 0.004 & 0.004 & 0.004 & 0.004 & 0.004 & 0.004 & 0.005 & 0.005 & 0.005 & 0.004 & 0.004 & 0.004 & 0.004 & 0.004 & 0.004 & 0.004 \\
\hline 0.80 & BIAS & 0.001 & -0.005 & 0.000 & -0.005 & -0.002 & 0.000 & 0.000 & 0.000 & 0.000 & 0.004 & -0.005 & -0.005 & -0.005 & -0.002 & 0.000 & 0.000 & 0.000 & 0.000 & 0.004 & 0.000 \\
\hline 0.80 & MSE & 0.002 & 0.002 & 0.002 & 0.002 & 0.002 & 0.002 & 0.002 & 0.002 & 0.002 & 0.002 & 0.002 & 0.002 & 0.002 & 0.002 & 0.002 & 0.002 & 0.002 & 0.002 & 0.002 & 0.002 \\
\hline 0.90 & BIAS & & -0.003 & & -0.003 & -0.001 & & & & 0.000 & & -0.003 & & & -0.001 & & 0.000 & & & 0.002 & 0.000 \\
\hline 0.90 & MSE & 0.001 & 0.001 & 0.001 & 0.001 & 0.001 & 0.001 & 0.001 & 0.001 & 0.001 & 0.001 & 0.001 & 0.001 & 0.001 & 0.001 & 0.001 & 0.001 & 0.001 & 0.001 & 0.001 & 0.001 \\
\hline
\end{tabular}


Table S18: Results for sample size $N=60$ and number of predictors $p=5$.

\begin{tabular}{|c|c|c|c|c|c|c|c|c|c|c|c|c|c|c|c|c|c|c|c|c|c|}
\hline$\rho^{2}$ & What & $R^{2}$ & $\mathrm{E}$ & OPE & $\mathrm{S}$ & $\mathrm{W}$ & OP1 & OP2 & OP5 & $\mathrm{P}$ & C & ML & $\mathrm{S}^{+}$ & $\mathrm{E}^{+}$ & $\mathrm{W}^{+}$ & $\mathrm{OP} 1^{+}$ & $\mathrm{OP} 2^{+}$ & $\mathrm{OP}^{+}$ & $\mathrm{P}^{+}$ & $\mathrm{C}^{+}$ & $\mathrm{OPE}^{+}$ \\
\hline 0.00 & BIAS & 0.085 & 0.000 & 0.000 & 0.002 & 0.018 & 0.002 & 0.000 & 0.000 & 0.000 & 0.018 & 0.025 & 0.022 & 0.022 & 0.030 & 0.023 & 0.023 & 0.022 & 0.022 & 0.031 & 0.022 \\
\hline 0.00 & MSE & 0.010 & 0.003 & 0.003 & 0.003 & 0.003 & 0.003 & 0.003 & 0.003 & 0.003 & 0.003 & 0.002 & 0.002 & 0.002 & 0.003 & 0.002 & 0.002 & 0.002 & 0.002 & 0.003 & 0.002 \\
\hline 0.01 & BIAS & 0.084 & 0.000 & 0.000 & 0.001 & 0.018 & 0.002 & 0.000 & 0.000 & 0.000 & 0.018 & 0.022 & 0.019 & 0.018 & 0.028 & 0.020 & 0.019 & 0.019 & 0.019 & 0.028 & 0.019 \\
\hline 0.01 & MSE & 0.010 & 0.004 & 0.004 & 0.004 & 0.004 & 0.004 & 0.004 & 0.004 & 0.004 & 0.004 & 0.003 & 0.002 & 0.002 & 0.003 & 0.003 & 0.002 & 0.002 & 0.002 & 0.003 & 0.002 \\
\hline 0.05 & BIAS & 0.079 & -0.001 & 0.000 & 0.000 & 0.016 & 0.002 & 0.000 & 0.000 & 0.000 & 0.017 & 0.014 & 0.009 & 0.008 & 0.020 & 0.011 & 0.010 & 0.010 & 0.010 & 0.022 & 0.010 \\
\hline 0.05 & MSE & 0.011 & 0.006 & 0.006 & 0.006 & 0.006 & 0.006 & 0.006 & 0.006 & 0.006 & 0.006 & 0.005 & 0.004 & 0.004 & 0.005 & 0.005 & 0.005 & 0.005 & 0.005 & 0.005 & 0.005 \\
\hline 0.10 & BIAS & 0.074 & -0.003 & 0.000 & -0.001 & 0.014 & 0.002 & 0.000 & 0.000 & 0.000 & 0.016 & 0.009 & 0.002 & 0.001 & 0.015 & 0.005 & 0.004 & 0.004 & 0.004 & 0.018 & 0.004 \\
\hline 0.10 & MSE & 0.012 & 0.008 & 0.008 & 0.008 & 0.008 & 0.008 & 0.008 & 0.008 & 0.008 & 0.008 & 0.007 & 0.007 & 0.007 & 0.007 & 0.007 & 0.007 & 0.007 & 0.007 & 0.008 & 0.007 \\
\hline 0.20 & BIAS & 0.063 & -0.005 & 0.000 & -0.004 & 0.009 & 0.001 & 0.000 & 0.000 & 0.000 & 0.014 & 0.005 & -0.004 & -0.005 & 0.010 & 0.002 & 0.001 & 0.001 & 0.000 & 0.014 & 0.001 \\
\hline 0.20 & MSE & 0.013 & 0.010 & 0.011 & 0.010 & 0.010 & 0.011 & 0.011 & 0.011 & 0.011 & 0.010 & 0.010 & 0.010 & 0.010 & 0.010 & 0.010 & 0.011 & 0.011 & 0.010 & 0.010 & 0.011 \\
\hline 0.30 & BIAS & 0.053 & -0.007 & 0.000 & -0.006 & 0.006 & 0.001 & 0.000 & 0.000 & 0.000 & 0.012 & 0.001 & -0.006 & -0.007 & 0.006 & 0.001 & 0.000 & 0.000 & 0.000 & 0.012 & 0.000 \\
\hline 0.30 & MSE & 0.012 & 0.011 & 0.012 & 0.011 & 0.011 & 0.011 & 0.012 & 0.012 & 0.012 & 0.011 & 0.011 & 0.011 & 0.011 & 0.011 & 0.011 & 0.012 & 0.012 & 0.012 & 0.011 & 0.012 \\
\hline 0.40 & BIAS & 0.044 & -0.008 & 0.000 & -0.007 & 0.003 & 0.001 & 0.000 & 0.000 & 0.000 & 0.010 & -0.001 & -0.007 & -0.008 & 0.003 & 0.001 & 0.000 & 0.000 & 0.000 & 0.010 & 0.000 \\
\hline 0.40 & MSE & 0.011 & 0.011 & 0.011 & 0.011 & 0.010 & 0.011 & 0.011 & 0.011 & 0.011 & 0.011 & 0.010 & 0.011 & 0.011 & 0.010 & 0.011 & 0.011 & 0.011 & 0.011 & 0.011 & 0.011 \\
\hline 0.50 & BIAS & 0.035 & -0.008 & 0.000 & -0.008 & 0.001 & 0.000 & 0.000 & 0.000 & 0.000 & 0.009 & -0.003 & -0.008 & -0.008 & 0.001 & 0.000 & 0.000 & 0.000 & 0.000 & 0.009 & 0.000 \\
\hline 0.50 & MSE & 0.009 & 0.009 & 0.009 & 0.009 & 0.009 & 0.009 & 0.009 & 0.009 & 0.009 & 0.009 & 0.009 & 0.009 & 0.009 & 0.009 & 0.009 & 0.009 & 0.009 & 0.009 & 0.009 & 0.009 \\
\hline 0.60 & BIAS & 0.027 & -0.008 & 0.000 & -0.007 & -0.001 & 0.000 & 0.000 & 0.000 & 0.000 & 0.007 & -0.005 & -0.007 & -0.008 & -0.001 & 0.000 & 0.000 & 0.000 & 0.000 & 0.007 & 0.000 \\
\hline 0.60 & MSE & 0.007 & 0.007 & 0.007 & 0.007 & 0.007 & 0.007 & 0.007 & 0.007 & 0.007 & 0.007 & 0.007 & 0.007 & 0.007 & 0.007 & 0.007 & 0.007 & 0.007 & 0.007 & 0.007 & 0.007 \\
\hline 0.70 & BIAS & 0.019 & -0.007 & 0.000 & -0.007 & -0.002 & 0.000 & 0.000 & 0.000 & 0.000 & 0.005 & -0.005 & -0.007 & -0.007 & -0.002 & 0.000 & 0.000 & 0.000 & 0.000 & 0.005 & 0.000 \\
\hline 0.70 & MSE & 0.004 & 0.005 & 0.005 & 0.005 & 0.005 & 0.005 & 0.005 & 0.005 & 0.005 & 0.005 & 0.005 & 0.005 & 0.005 & 0.005 & 0.005 & 0.005 & 0.005 & 0.005 & 0.005 & 0.005 \\
\hline 0.80 & BIAS & 0.012 & -0.005 & 0.000 & -0.005 & -0.002 & 0.000 & 0.000 & 0.000 & 0.000 & 0.004 & -0.004 & -0.005 & -0.005 & -0.002 & 0.000 & 0.000 & 0.000 & 0.000 & 0.004 & 0.000 \\
\hline 0.80 & MSE & 0.002 & 0.003 & 0.002 & 0.003 & 0.002 & 0.002 & 0.002 & 0.002 & 0.002 & 0.002 & 0.002 & 0.003 & 0.003 & 0.002 & 0.002 & 0.002 & 0.002 & 0.002 & 0.002 & 0.002 \\
\hline 0.90 & BIAS & & -0.003 & & -0.003 & -0.001 & & & & 0.000 & & & & & -0.001 & & 0.000 & & & 0.002 & 0.000 \\
\hline 0.90 & MSE & 0.001 & 0.001 & 0.001 & 0.001 & 0.001 & 0.001 & 0.001 & 0.001 & 0.001 & 0.001 & 0.001 & 0.001 & 0.001 & 0.001 & 0.001 & 0.001 & 0.001 & 0.001 & 0.001 & 0.001 \\
\hline
\end{tabular}


Table S19: Results for sample size $N=60$ and number of predictors $p=10$.

\begin{tabular}{|c|c|c|c|c|c|c|c|c|c|c|c|c|c|c|c|c|c|c|c|c|c|}
\hline$\rho^{2}$ & What & $R^{2}$ & $\mathrm{E}$ & $\mathrm{OPE}$ & $\mathrm{S}$ & $\mathrm{W}$ & OP1 & OP2 & OP5 & $\mathrm{P}$ & C & ML & $\mathrm{S}^{+}$ & $\mathrm{E}^{+}$ & $\mathrm{W}^{+}$ & $\mathrm{OP} 1^{+}$ & $\mathrm{OP} 2^{+}$ & $\mathrm{OP}^{+}$ & $\mathrm{P}^{+}$ & $\mathrm{C}^{+}$ & $\mathrm{OPE}^{+}$ \\
\hline 0.00 & BIAS & 0.170 & 0.000 & 0.000 & 0.004 & 0.020 & 0.003 & 0.001 & 0.000 & 0.001 & 0.018 & 0.037 & 0.034 & 0.033 & 0.042 & 0.035 & 0.034 & 0.034 & 0.034 & 0.042 & 0.034 \\
\hline 0.00 & MSE & 0.033 & 0.007 & 0.007 & 0.007 & 0.007 & 0.007 & 0.007 & 0.007 & 0.007 & 0.007 & 0.005 & 0.004 & 0.004 & 0.005 & 0.004 & 0.004 & 0.004 & 0.004 & 0.005 & 0.004 \\
\hline 0.01 & BIAS & 0.168 & 0.000 & 0.000 & 0.003 & 0.020 & 0.003 & 0.001 & 0.000 & 0.000 & 0.018 & 0.033 & 0.030 & 0.029 & 0.039 & 0.031 & 0.030 & 0.030 & 0.030 & 0.039 & 0.030 \\
\hline 0.01 & MSE & 0.033 & 0.007 & 0.008 & 0.007 & 0.007 & 0.008 & 0.008 & 0.008 & 0.008 & 0.008 & 0.005 & 0.004 & 0.004 & 0.006 & 0.005 & 0.005 & 0.005 & 0.005 & 0.006 & 0.005 \\
\hline 0.05 & BIAS & 0.160 & -0.001 & 0.000 & 0.002 & 0.018 & 0.002 & 0.001 & 0.000 & 0.000 & 0.017 & 0.021 & 0.018 & 0.016 & 0.029 & 0.019 & 0.018 & 0.018 & 0.018 & 0.029 & 0.018 \\
\hline 0.05 & MSE & 0.032 & 0.009 & 0.009 & 0.009 & 0.009 & 0.009 & 0.009 & 0.009 & 0.009 & 0.009 & 0.007 & 0.006 & 0.006 & 0.007 & 0.006 & 0.006 & 0.006 & 0.006 & 0.007 & 0.006 \\
\hline 0.10 & BIAS & 0.150 & -0.003 & 0.000 & 0.000 & 0.015 & 0.002 & 0.001 & 0.000 & 0.000 & 0.016 & 0.013 & 0.008 & 0.005 & 0.020 & 0.010 & 0.009 & 0.008 & 0.008 & 0.021 & 0.008 \\
\hline 0.10 & MSE & 0.030 & 0.011 & 0.011 & 0.011 & 0.010 & 0.011 & 0.011 & 0.011 & 0.011 & 0.011 & 0.009 & 0.009 & 0.009 & 0.009 & 0.009 & 0.009 & 0.009 & 0.009 & 0.010 & 0.009 \\
\hline 0.20 & BIAS & 0.131 & -0.005 & 0.000 & -0.002 & 0.011 & 0.002 & 0.000 & 0.000 & 0.000 & 0.014 & 0.005 & -0.001 & -0.004 & 0.012 & 0.003 & 0.002 & 0.002 & 0.001 & 0.015 & 0.002 \\
\hline 0.20 & MSE & 0.026 & 0.013 & 0.013 & 0.013 & 0.012 & 0.013 & 0.013 & 0.013 & 0.013 & 0.013 & 0.012 & 0.012 & 0.012 & 0.012 & 0.013 & 0.013 & 0.013 & 0.013 & 0.013 & 0.013 \\
\hline 0.30 & BIAS & 0.113 & -0.007 & 0.000 & -0.004 & 0.007 & 0.001 & 0.000 & 0.000 & 0.000 & 0.012 & 0.002 & -0.004 & -0.007 & 0.007 & 0.001 & 0.000 & 0.000 & 0.000 & 0.012 & 0.000 \\
\hline 0.30 & MSE & 0.022 & 0.013 & 0.013 & 0.013 & 0.013 & 0.013 & 0.013 & 0.013 & 0.013 & 0.013 & 0.013 & 0.013 & 0.013 & 0.013 & 0.013 & 0.013 & 0.013 & 0.013 & 0.013 & 0.013 \\
\hline 0.40 & BIAS & 0.095 & -0.008 & 0.000 & -0.006 & 0.004 & 0.001 & 0.000 & 0.000 & 0.000 & 0.010 & -0.001 & -0.006 & -0.008 & 0.004 & 0.001 & 0.000 & 0.000 & 0.000 & 0.011 & 0.000 \\
\hline 0.40 & MSE & 0.017 & 0.012 & 0.012 & 0.012 & 0.012 & 0.012 & 0.012 & 0.012 & 0.012 & 0.012 & 0.012 & 0.012 & 0.012 & 0.012 & 0.012 & 0.012 & 0.012 & 0.012 & 0.012 & 0.012 \\
\hline 0.50 & BIAS & 0.078 & -0.008 & 0.000 & -0.006 & 0.002 & 0.001 & 0.000 & 0.000 & 0.000 & 0.009 & -0.003 & -0.006 & -0.008 & 0.002 & 0.001 & 0.000 & 0.000 & 0.000 & 0.009 & 0.000 \\
\hline 0.50 & MSE & 0.013 & 0.010 & 0.010 & 0.010 & 0.010 & 0.010 & 0.010 & 0.010 & 0.010 & 0.010 & 0.010 & 0.010 & 0.010 & 0.010 & 0.010 & 0.010 & 0.010 & 0.010 & 0.010 & 0.010 \\
\hline 0.60 & BIAS & 0.061 & -0.008 & 0.000 & -0.007 & 0.000 & 0.000 & 0.000 & 0.000 & 0.000 & 0.007 & -0.004 & -0.007 & -0.008 & 0.000 & 0.000 & 0.000 & 0.000 & 0.000 & 0.007 & 0.000 \\
\hline 0.60 & MSE & 0.009 & 0.008 & 0.008 & 0.008 & 0.008 & 0.008 & 0.008 & 0.008 & 0.008 & 0.008 & 0.008 & 0.008 & 0.008 & 0.008 & 0.008 & 0.008 & 0.008 & 0.008 & 0.008 & 0.008 \\
\hline 0.70 & BIAS & 0.045 & -0.007 & 0.000 & -0.006 & -0.001 & 0.000 & 0.000 & 0.000 & 0.000 & 0.005 & -0.005 & -0.006 & -0.007 & -0.001 & 0.000 & 0.000 & 0.000 & 0.000 & 0.005 & 0.000 \\
\hline 0.70 & MSE & 0.006 & 0.005 & 0.005 & 0.005 & 0.005 & 0.005 & 0.005 & 0.005 & 0.005 & 0.005 & 0.005 & 0.005 & 0.005 & 0.005 & 0.005 & 0.005 & 0.005 & 0.005 & 0.005 & 0.005 \\
\hline 0.80 & BIAS & 0.029 & -0.005 & 0.000 & -0.005 & -0.001 & 0.000 & 0.000 & 0.000 & 0.000 & 0.004 & -0.004 & -0.005 & -0.005 & -0.001 & 0.000 & 0.000 & 0.000 & 0.000 & 0.004 & 0.000 \\
\hline 0.80 & MSE & 0.003 & 0.003 & 0.003 & 0.003 & 0.003 & 0.003 & 0.003 & 0.003 & 0.003 & 0.002 & 0.003 & 0.003 & 0.003 & 0.003 & 0.003 & 0.003 & 0.003 & 0.003 & 0.002 & 0.003 \\
\hline 0.90 & BIAS & & -0.003 & & -0.003 & -0.001 & & & & 0.000 & & & & & -0.001 & & 0.000 & & & 0.002 & 0.000 \\
\hline 0.90 & MSE & 0.001 & 0.001 & 0.001 & 0.001 & 0.001 & 0.001 & 0.001 & 0.001 & 0.001 & 0.001 & 0.001 & 0.001 & 0.001 & 0.001 & 0.001 & 0.001 & 0.001 & 0.001 & 0.001 & 0.001 \\
\hline
\end{tabular}


Table S20: Results for sample size $N=100$ and number of predictors $p=2$.

\begin{tabular}{|c|c|c|c|c|c|c|c|c|c|c|c|c|c|c|c|c|c|c|c|c|c|}
\hline$\rho^{2}$ & What & $R^{2}$ & $\mathrm{E}$ & OPE & $\mathrm{S}$ & W & OP1 & $\mathrm{OP} 2$ & OP5 & $\mathrm{P}$ & C & ML & $\mathrm{S}^{+}$ & $\mathrm{E}^{+}$ & $\mathrm{W}^{+}$ & $\mathrm{OP} 1^{+}$ & $\mathrm{OP} 2^{+}$ & $\mathrm{OP}^{+}$ & $\mathrm{P}^{+}$ & $\mathrm{C}^{+}$ & $\mathrm{OPE}^{+}$ \\
\hline 0.00 & BIAS & 0.020 & 0.000 & 0.000 & 0.000 & 0.010 & 0.001 & 0.000 & 0.000 & 0.000 & 0.010 & 0.010 & 0.008 & 0.008 & 0.012 & 0.008 & 0.008 & 0.008 & 0.008 & 0.013 & 0.008 \\
\hline 0.00 & MSE & 0.001 & 0.000 & 0.000 & 0.000 & 0.001 & 0.000 & 0.000 & 0.000 & 0.000 & 0.001 & 0.000 & 0.000 & 0.000 & 0.000 & 0.000 & 0.000 & 0.000 & 0.000 & 0.001 & 0.000 \\
\hline 0.01 & BIAS & 0.020 & 0.000 & 0.000 & 0.000 & 0.010 & 0.001 & 0.000 & 0.000 & 0.000 & 0.010 & 0.009 & 0.005 & 0.005 & 0.011 & 0.006 & 0.005 & 0.005 & 0.005 & 0.012 & 0.005 \\
\hline 0.01 & MSE & 0.001 & 0.001 & 0.001 & 0.001 & 0.001 & 0.001 & 0.001 & 0.001 & 0.001 & 0.001 & 0.001 & 0.001 & 0.001 & 0.001 & 0.001 & 0.001 & 0.001 & 0.001 & 0.001 & 0.001 \\
\hline 0.05 & BIAS & 0.018 & -0.001 & 0.000 & -0.001 & 0.009 & 0.001 & 0.000 & 0.000 & 0.000 & 0.010 & 0.007 & 0.000 & 0.000 & 0.009 & 0.002 & 0.001 & 0.001 & 0.001 & 0.010 & 0.001 \\
\hline 0.05 & MSE & 0.002 & 0.002 & 0.002 & 0.002 & 0.002 & 0.002 & 0.002 & 0.002 & 0.002 & 0.002 & 0.002 & 0.002 & 0.002 & 0.002 & 0.002 & 0.002 & 0.002 & 0.002 & 0.002 & 0.002 \\
\hline 0.10 & BIAS & 0.016 & -0.002 & 0.000 & -0.002 & 0.007 & 0.001 & 0.000 & 0.000 & 0.000 & 0.009 & 0.006 & -0.001 & -0.002 & 0.007 & 0.001 & 0.000 & 0.000 & 0.000 & 0.009 & 0.000 \\
\hline 0.10 & MSE & 0.004 & 0.003 & 0.004 & 0.003 & 0.003 & 0.004 & 0.004 & 0.004 & 0.004 & 0.004 & 0.003 & 0.003 & 0.003 & 0.003 & 0.004 & 0.004 & 0.004 & 0.004 & 0.004 & 0.004 \\
\hline 0.20 & BIAS & 0.013 & -0.003 & 0.000 & -0.003 & 0.005 & 0.000 & 0.000 & 0.000 & 0.000 & 0.008 & 0.003 & -0.003 & -0.003 & 0.005 & 0.000 & 0.000 & 0.000 & 0.000 & 0.008 & 0.000 \\
\hline 0.20 & MSE & 0.005 & 0.005 & 0.005 & 0.005 & 0.005 & 0.005 & 0.005 & 0.005 & 0.005 & 0.005 & 0.005 & 0.005 & 0.005 & 0.005 & 0.005 & 0.005 & 0.005 & 0.005 & 0.005 & 0.005 \\
\hline 0.30 & BIAS & 0.010 & -0.004 & 0.000 & -0.004 & 0.003 & 0.000 & 0.000 & 0.000 & 0.000 & 0.007 & 0.001 & -0.004 & -0.004 & 0.003 & 0.000 & 0.000 & 0.000 & 0.000 & 0.007 & 0.000 \\
\hline 0.30 & MSE & 0.006 & 0.006 & 0.006 & 0.006 & 0.006 & 0.006 & 0.006 & 0.006 & 0.006 & 0.006 & 0.006 & 0.006 & 0.006 & 0.006 & 0.006 & 0.006 & 0.006 & 0.006 & 0.006 & 0.006 \\
\hline 0.40 & BIAS & 0.007 & -0.005 & 0.000 & -0.005 & 0.001 & 0.000 & 0.000 & 0.000 & 0.000 & 0.006 & -0.001 & -0.005 & -0.005 & 0.001 & 0.000 & 0.000 & 0.000 & 0.000 & 0.006 & 0.000 \\
\hline 0.40 & MSE & 0.006 & 0.006 & 0.006 & 0.006 & 0.006 & 0.006 & 0.006 & 0.006 & 0.006 & 0.006 & 0.006 & 0.006 & 0.006 & 0.006 & 0.006 & 0.006 & 0.006 & 0.006 & 0.006 & 0.006 \\
\hline 0.50 & BIAS & 0.005 & -0.005 & 0.000 & -0.005 & 0.000 & 0.000 & 0.000 & 0.000 & 0.000 & 0.005 & -0.002 & -0.005 & -0.005 & 0.000 & 0.000 & 0.000 & 0.000 & 0.000 & 0.005 & 0.000 \\
\hline 0.50 & MSE & 0.005 & 0.005 & 0.005 & 0.005 & 0.005 & 0.005 & 0.005 & 0.005 & 0.005 & 0.005 & 0.005 & 0.005 & 0.005 & 0.005 & 0.005 & 0.005 & 0.005 & 0.005 & 0.005 & 0.005 \\
\hline 0.60 & BIAS & 0.003 & -0.005 & 0.000 & -0.005 & -0.001 & 0.000 & 0.000 & 0.000 & 0.000 & 0.004 & -0.003 & -0.005 & -0.005 & -0.001 & 0.000 & 0.000 & 0.000 & 0.000 & 0.004 & 0.000 \\
\hline 0.60 & MSE & 0.004 & 0.004 & 0.004 & 0.004 & 0.004 & 0.004 & 0.004 & 0.004 & 0.004 & 0.004 & 0.004 & 0.004 & 0.004 & 0.004 & 0.004 & 0.004 & 0.004 & 0.004 & 0.004 & 0.004 \\
\hline 0.70 & BIAS & 0.002 & -0.004 & 0.000 & -0.004 & -0.001 & 0.000 & 0.000 & 0.000 & 0.000 & 0.003 & -0.003 & -0.004 & -0.004 & -0.001 & 0.000 & 0.000 & 0.000 & 0.000 & 0.003 & 0.000 \\
\hline 0.70 & MSE & 0.003 & 0.003 & 0.003 & 0.003 & 0.003 & 0.003 & 0.003 & 0.003 & 0.003 & 0.003 & 0.003 & 0.003 & 0.003 & 0.003 & 0.003 & 0.003 & 0.003 & 0.003 & 0.003 & 0.003 \\
\hline 0.80 & BIAS & 0.001 & -0.003 & 0.000 & -0.003 & -0.001 & 0.000 & 0.000 & 0.000 & 0.000 & 0.002 & -0.003 & -0.003 & -0.003 & -0.001 & 0.000 & 0.000 & 0.000 & 0.000 & 0.002 & 0.000 \\
\hline 0.80 & MSE & 0.001 & 0.001 & 0.001 & 0.001 & 0.001 & 0.001 & 0.001 & 0.001 & 0.001 & 0.001 & 0.001 & 0.001 & 0.001 & 0.001 & 0.001 & 0.001 & 0.001 & 0.001 & 0.001 & 0.001 \\
\hline 0.90 & BIAS & 0.000 & -0.002 & 0.000 & -0.002 & -0.001 & 0.000 & 0.000 & 0.000 & 0.000 & 0.001 & -0.002 & -0.002 & -0.002 & -0.001 & 0.000 & 0.000 & 0.000 & 0.000 & 0.001 & 0.000 \\
\hline 0.90 & MSE & 0.000 & 0.000 & 0.000 & 0.000 & 0.000 & 0.000 & 0.000 & 0.000 & 0.000 & 0.000 & 0.000 & 0.000 & 0.000 & 0.000 & 0.000 & 0.000 & 0.000 & 0.000 & 0.000 & 0.000 \\
\hline
\end{tabular}


Table S21: Results for sample size $N=100$ and number of predictors $p=5$.

\begin{tabular}{|c|c|c|c|c|c|c|c|c|c|c|c|c|c|c|c|c|c|c|c|c|c|}
\hline$\rho^{2}$ & What & $R^{2}$ & $\mathrm{E}$ & OPE & $\mathrm{S}$ & W & OP1 & $\mathrm{OP} 2$ & OP5 & $\mathrm{P}$ & C & ML & $\mathrm{S}^{+}$ & $\mathrm{E}^{+}$ & $\mathrm{w}^{+}$ & $\mathrm{OP} 1^{+}$ & $\mathrm{OP} 2^{+}$ & $\mathrm{OP}^{+}$ & $\mathrm{P}^{+}$ & $\mathrm{C}^{+}$ & $\mathrm{OPE}^{+}$ \\
\hline 0.00 & BIAS & 0.050 & 0.000 & 0.000 & 0.000 & 0.010 & 0.001 & 0.000 & 0.000 & 0.000 & 0.010 & 0.015 & 0.013 & 0.013 & 0.018 & 0.013 & 0.013 & 0.013 & 0.013 & 0.018 & 0.013 \\
\hline 0.00 & MSE & 0.003 & 0.001 & 0.001 & 0.001 & 0.001 & 0.001 & 0.001 & 0.001 & 0.001 & 0.001 & 0.001 & 0.001 & 0.001 & 0.001 & 0.001 & 0.001 & 0.001 & 0.001 & 0.001 & 0.001 \\
\hline 0.01 & BIAS & 0.050 & 0.000 & 0.000 & 0.000 & 0.010 & 0.001 & 0.000 & 0.000 & 0.000 & 0.010 & 0.012 & 0.010 & 0.009 & 0.015 & 0.010 & 0.010 & 0.010 & 0.010 & 0.016 & 0.010 \\
\hline 0.01 & MSE & 0.004 & 0.001 & 0.001 & 0.001 & 0.001 & 0.001 & 0.001 & 0.001 & 0.001 & 0.002 & 0.001 & 0.001 & 0.001 & 0.001 & 0.001 & 0.001 & 0.001 & 0.001 & 0.001 & 0.001 \\
\hline 0.05 & BIAS & 0.047 & -0.001 & 0.000 & 0.000 & 0.009 & 0.001 & 0.000 & 0.000 & 0.000 & 0.010 & 0.007 & 0.002 & 0.002 & 0.011 & 0.004 & 0.003 & 0.003 & 0.003 & 0.011 & 0.003 \\
\hline 0.05 & MSE & 0.005 & 0.003 & 0.003 & 0.003 & 0.003 & 0.003 & 0.003 & 0.003 & 0.003 & 0.003 & 0.003 & 0.002 & 0.002 & 0.003 & 0.002 & 0.002 & 0.002 & 0.002 & 0.003 & 0.002 \\
\hline 0.10 & BIAS & 0.044 & -0.002 & 0.000 & -0.001 & 0.008 & 0.001 & 0.000 & 0.000 & 0.000 & 0.009 & 0.005 & -0.001 & -0.001 & 0.008 & 0.001 & 0.001 & 0.001 & 0.001 & 0.010 & 0.001 \\
\hline 0.10 & MSE & 0.006 & 0.004 & 0.004 & 0.004 & 0.004 & 0.004 & 0.004 & 0.004 & 0.004 & 0.004 & 0.004 & 0.004 & 0.004 & 0.004 & 0.004 & 0.004 & 0.004 & 0.004 & 0.004 & 0.004 \\
\hline 0.20 & BIAS & 0.038 & -0.003 & 0.000 & -0.003 & 0.005 & 0.001 & 0.000 & 0.000 & 0.000 & 0.008 & 0.003 & -0.003 & -0.003 & 0.005 & 0.001 & 0.000 & 0.000 & 0.000 & 0.008 & 0.000 \\
\hline 0.20 & MSE & 0.007 & 0.006 & 0.006 & 0.006 & 0.006 & 0.006 & 0.006 & 0.006 & 0.006 & 0.006 & 0.006 & 0.006 & 0.006 & 0.006 & 0.006 & 0.006 & 0.006 & 0.006 & 0.006 & 0.006 \\
\hline 0.30 & BIAS & 0.032 & -0.004 & 0.000 & -0.004 & 0.003 & 0.001 & 0.000 & 0.000 & 0.000 & 0.007 & 0.001 & -0.004 & -0.004 & 0.003 & 0.001 & 0.000 & 0.000 & 0.000 & 0.007 & 0.000 \\
\hline 0.30 & MSE & 0.007 & 0.006 & 0.006 & 0.006 & 0.006 & 0.006 & 0.006 & 0.006 & 0.006 & 0.006 & 0.006 & 0.006 & 0.006 & 0.006 & 0.006 & 0.006 & 0.006 & 0.006 & 0.006 & 0.006 \\
\hline 0.40 & BIAS & 0.026 & -0.005 & 0.000 & -0.004 & 0.002 & 0.000 & 0.000 & 0.000 & 0.000 & 0.006 & -0.001 & -0.004 & -0.005 & 0.002 & 0.000 & 0.000 & 0.000 & 0.000 & 0.006 & 0.000 \\
\hline 0.40 & MSE & 0.006 & 0.006 & 0.006 & 0.006 & 0.006 & 0.006 & 0.006 & 0.006 & 0.006 & 0.006 & 0.006 & 0.006 & 0.006 & 0.006 & 0.006 & 0.006 & 0.006 & 0.006 & 0.006 & 0.006 \\
\hline 0.50 & BIAS & 0.021 & -0.005 & 0.000 & -0.005 & 0.000 & 0.000 & 0.000 & 0.000 & 0.000 & 0.005 & -0.002 & -0.005 & -0.005 & 0.000 & 0.000 & 0.000 & 0.000 & 0.000 & 0.005 & 0.000 \\
\hline 0.50 & MSE & 0.005 & 0.005 & 0.005 & 0.005 & 0.005 & 0.005 & 0.005 & 0.005 & 0.005 & 0.005 & 0.005 & 0.005 & 0.005 & 0.005 & 0.005 & 0.005 & 0.005 & 0.005 & 0.005 & 0.005 \\
\hline 0.60 & BIAS & 0.016 & -0.005 & 0.000 & -0.005 & 0.000 & 0.000 & 0.000 & 0.000 & 0.000 & 0.004 & -0.003 & -0.005 & -0.005 & 0.000 & 0.000 & 0.000 & 0.000 & 0.000 & 0.004 & 0.000 \\
\hline 0.60 & MSE & 0.004 & 0.004 & 0.004 & 0.004 & 0.004 & 0.004 & 0.004 & 0.004 & 0.004 & 0.004 & 0.004 & 0.004 & 0.004 & 0.004 & 0.004 & 0.004 & 0.004 & 0.004 & 0.004 & 0.004 \\
\hline 0.70 & BIAS & 0.011 & -0.004 & 0.000 & -0.004 & -0.001 & 0.000 & 0.000 & 0.000 & 0.000 & 0.003 & -0.003 & -0.004 & -0.004 & -0.001 & 0.000 & 0.000 & 0.000 & 0.000 & 0.003 & 0.000 \\
\hline 0.70 & MSE & 0.003 & 0.003 & 0.003 & 0.003 & 0.003 & 0.003 & 0.003 & 0.003 & 0.003 & 0.003 & 0.003 & 0.003 & 0.003 & 0.003 & 0.003 & 0.003 & 0.003 & 0.003 & 0.003 & 0.003 \\
\hline 0.80 & BIAS & 0.007 & -0.003 & 0.000 & -0.003 & -0.001 & 0.000 & 0.000 & 0.000 & 0.000 & 0.002 & -0.003 & -0.003 & -0.003 & -0.001 & 0.000 & 0.000 & 0.000 & 0.000 & 0.002 & 0.000 \\
\hline 0.80 & MSE & 0.001 & 0.001 & 0.001 & 0.001 & 0.001 & 0.001 & 0.001 & 0.001 & 0.001 & 0.001 & 0.001 & 0.001 & 0.001 & 0.001 & 0.001 & 0.001 & 0.001 & 0.001 & 0.001 & 0.001 \\
\hline 0.90 & BIAS & 0.003 & -0.002 & 0.000 & -0.002 & -0.001 & 0.000 & 0.000 & 0.000 & 0.000 & 0.001 & -0.002 & -0.002 & -0.002 & -0.001 & 0.000 & 0.000 & 0.000 & 0.000 & 0.001 & 0.000 \\
\hline 0.90 & MSE & 0.000 & 0.000 & 0.000 & 0.000 & 0.000 & 0.000 & 0.000 & 0.000 & 0.000 & 0.000 & 0.000 & 0.000 & 0.000 & 0.000 & 0.000 & 0.000 & 0.000 & 0.000 & 0.000 & 0.000 \\
\hline
\end{tabular}


Table S22: Results for sample size $N=100$ and number of predictors $p=10$.

\begin{tabular}{|c|c|c|c|c|c|c|c|c|c|c|c|c|c|c|c|c|c|c|c|c|c|}
\hline$\rho^{2}$ & What & $R^{2}$ & $\mathrm{E}$ & OPE & $\mathrm{S}$ & $\mathrm{W}$ & OP1 & OP2 & OP5 & $\mathrm{P}$ & C & ML & $\mathrm{S}^{+}$ & $\mathrm{E}^{+}$ & $\mathrm{W}^{+}$ & $\mathrm{OP} 1^{+}$ & $\mathrm{OP} 2^{+}$ & $\mathrm{OP}^{+}$ & $\mathrm{P}^{+}$ & $\mathrm{C}^{+}$ & $\mathrm{OPE}^{+}$ \\
\hline 0.00 & BIAS & 0.101 & 0.000 & 0.000 & 0.001 & 0.011 & 0.001 & 0.000 & 0.000 & 0.000 & 0.010 & 0.021 & 0.019 & 0.019 & 0.024 & 0.020 & 0.019 & 0.019 & 0.019 & 0.024 & 0.019 \\
\hline 0.00 & MSE & 0.012 & 0.002 & 0.002 & 0.002 & 0.002 & 0.002 & 0.002 & 0.002 & 0.002 & 0.002 & 0.002 & 0.001 & 0.001 & 0.002 & 0.001 & 0.001 & 0.001 & 0.001 & 0.002 & 0.001 \\
\hline 0.01 & BIAS & 0.100 & 0.000 & 0.000 & 0.001 & 0.011 & 0.001 & 0.000 & 0.000 & 0.000 & 0.010 & 0.017 & 0.016 & 0.015 & 0.021 & 0.016 & 0.016 & 0.016 & 0.016 & 0.021 & 0.016 \\
\hline 0.01 & MSE & 0.012 & 0.003 & 0.003 & 0.003 & 0.003 & 0.003 & 0.003 & 0.003 & 0.003 & 0.003 & 0.002 & 0.002 & 0.002 & 0.002 & 0.002 & 0.002 & 0.002 & 0.002 & 0.002 & 0.002 \\
\hline 0.05 & BIAS & 0.095 & -0.001 & 0.000 & 0.000 & 0.010 & 0.001 & 0.000 & 0.000 & 0.000 & 0.010 & 0.009 & 0.006 & 0.005 & 0.014 & 0.007 & 0.006 & 0.006 & 0.006 & 0.014 & 0.006 \\
\hline 0.05 & MSE & 0.012 & 0.004 & 0.004 & 0.004 & 0.004 & 0.004 & 0.004 & 0.004 & 0.004 & 0.004 & 0.003 & 0.003 & 0.003 & 0.003 & 0.003 & 0.003 & 0.003 & 0.003 & 0.003 & 0.003 \\
\hline 0.10 & BIAS & 0.090 & -0.001 & 0.000 & 0.000 & 0.009 & 0.001 & 0.001 & 0.000 & 0.000 & 0.010 & 0.005 & 0.001 & 0.000 & 0.009 & 0.002 & 0.002 & 0.002 & 0.002 & 0.011 & 0.002 \\
\hline 0.10 & MSE & 0.012 & 0.005 & 0.005 & 0.005 & 0.005 & 0.005 & 0.005 & 0.005 & 0.005 & 0.005 & 0.005 & 0.005 & 0.005 & 0.005 & 0.005 & 0.005 & 0.005 & 0.005 & 0.005 & 0.005 \\
\hline 0.20 & BIAS & 0.078 & -0.003 & 0.001 & -0.002 & 0.006 & 0.001 & 0.001 & 0.001 & 0.001 & 0.009 & 0.003 & -0.002 & -0.003 & 0.006 & 0.001 & 0.001 & 0.001 & 0.001 & 0.009 & 0.001 \\
\hline 0.20 & MSE & 0.011 & 0.006 & 0.007 & 0.006 & 0.006 & 0.007 & 0.007 & 0.007 & 0.007 & 0.007 & 0.006 & 0.006 & 0.006 & 0.006 & 0.007 & 0.007 & 0.007 & 0.007 & 0.007 & 0.007 \\
\hline 0.30 & BIAS & 0.068 & -0.004 & 0.001 & -0.003 & 0.004 & 0.001 & 0.001 & 0.001 & 0.001 & 0.008 & 0.001 & -0.003 & -0.004 & 0.004 & 0.001 & 0.001 & 0.001 & 0.001 & 0.008 & 0.001 \\
\hline 0.30 & MSE & 0.010 & 0.007 & 0.007 & 0.007 & 0.007 & 0.007 & 0.007 & 0.007 & 0.007 & 0.007 & 0.007 & 0.007 & 0.007 & 0.007 & 0.007 & 0.007 & 0.007 & 0.007 & 0.007 & 0.007 \\
\hline 0.40 & BIAS & 0.057 & -0.004 & 0.001 & -0.003 & 0.003 & 0.001 & 0.001 & 0.001 & 0.001 & 0.007 & 0.000 & -0.003 & -0.004 & 0.003 & 0.001 & 0.001 & 0.001 & 0.001 & 0.007 & 0.001 \\
\hline 0.40 & MSE & 0.009 & 0.007 & 0.007 & 0.007 & 0.006 & 0.007 & 0.007 & 0.007 & 0.007 & 0.007 & 0.006 & 0.007 & 0.007 & 0.006 & 0.007 & 0.007 & 0.007 & 0.007 & 0.007 & 0.007 \\
\hline 0.50 & BIAS & 0.047 & -0.004 & 0.001 & -0.004 & 0.001 & 0.001 & 0.001 & 0.001 & 0.001 & 0.006 & -0.002 & -0.004 & -0.004 & 0.001 & 0.001 & 0.001 & 0.001 & 0.001 & 0.006 & 0.001 \\
\hline 0.50 & MSE & 0.007 & 0.006 & 0.006 & 0.006 & 0.006 & 0.006 & 0.006 & 0.006 & 0.006 & 0.006 & 0.006 & 0.006 & 0.006 & 0.006 & 0.006 & 0.006 & 0.006 & 0.006 & 0.006 & 0.006 \\
\hline 0.60 & BIAS & 0.037 & -0.004 & 0.001 & -0.004 & 0.000 & 0.001 & 0.001 & 0.001 & 0.000 & 0.005 & -0.002 & -0.004 & -0.004 & 0.000 & 0.001 & 0.001 & 0.001 & 0.000 & 0.005 & 0.001 \\
\hline 0.60 & MSE & 0.005 & 0.004 & 0.004 & 0.004 & 0.004 & 0.004 & 0.004 & 0.004 & 0.004 & 0.004 & 0.004 & 0.004 & 0.004 & 0.004 & 0.004 & 0.004 & 0.004 & 0.004 & 0.004 & 0.004 \\
\hline 0.70 & BIAS & 0.027 & -0.004 & 0.000 & -0.003 & 0.000 & 0.000 & 0.000 & 0.000 & 0.000 & 0.003 & -0.003 & -0.003 & -0.004 & 0.000 & 0.000 & 0.000 & 0.000 & 0.000 & 0.003 & 0.000 \\
\hline 0.70 & MSE & 0.003 & 0.003 & 0.003 & 0.003 & 0.003 & 0.003 & 0.003 & 0.003 & 0.003 & 0.003 & 0.003 & 0.003 & 0.003 & 0.003 & 0.003 & 0.003 & 0.003 & 0.003 & 0.003 & 0.003 \\
\hline 0.80 & BIAS & 0.018 & -0.003 & 0.000 & -0.003 & -0.001 & 0.000 & 0.000 & 0.000 & 0.000 & 0.002 & -0.002 & -0.003 & -0.003 & -0.001 & 0.000 & 0.000 & 0.000 & 0.000 & 0.002 & 0.000 \\
\hline 0.80 & MSE & 0.001 & 0.001 & 0.001 & 0.001 & 0.001 & 0.001 & 0.001 & 0.001 & 0.001 & 0.001 & 0.001 & 0.001 & 0.001 & 0.001 & 0.001 & 0.001 & 0.001 & 0.001 & 0.001 & 0.001 \\
\hline 0.90 & BIAS & & -0.002 & & -0.002 & -0.001 & & & & 0.000 & & & & -0.002 & -0.001 & & 0.000 & 0.000 & & 0.001 & 0.000 \\
\hline 0.90 & MSE & 0.000 & 0.000 & 0.000 & 0.000 & 0.000 & 0.000 & 0.000 & 0.000 & 0.000 & 0.000 & 0.000 & 0.000 & 0.000 & 0.000 & 0.000 & 0.000 & 0.000 & 0.000 & 0.000 & 0.000 \\
\hline
\end{tabular}


Table S23: Results for sample size $N=150$ and number of predictors $p=2$.

\begin{tabular}{|c|c|c|c|c|c|c|c|c|c|c|c|c|c|c|c|c|c|c|c|c|c|}
\hline$\rho^{2}$ & What & $R^{2}$ & $\mathrm{E}$ & OPE & $\mathrm{S}$ & W & OP1 & $\mathrm{OP} 2$ & OP5 & $\mathrm{P}$ & C & ML & $\mathrm{S}^{+}$ & $\mathrm{E}^{+}$ & $\mathrm{w}^{+}$ & $\mathrm{OP} 1^{+}$ & $\mathrm{OP} 2^{+}$ & $\mathrm{OP}^{+}$ & $\mathrm{P}^{+}$ & $\mathrm{C}^{+}$ & $\mathrm{OPE}^{+}$ \\
\hline 0.00 & BIAS & 0.013 & 0.000 & 0.000 & 0.000 & 0.007 & 0.000 & 0.000 & 0.000 & 0.000 & 0.007 & 0.006 & 0.005 & 0.005 & 0.008 & 0.005 & 0.005 & 0.005 & 0.005 & 0.008 & 0.005 \\
\hline 0.00 & MSE & 0.000 & 0.000 & 0.000 & 0.000 & 0.000 & 0.000 & 0.000 & 0.000 & 0.000 & 0.000 & 0.000 & 0.000 & 0.000 & 0.000 & 0.000 & 0.000 & 0.000 & 0.000 & 0.000 & 0.000 \\
\hline 0.01 & BIAS & 0.013 & 0.000 & 0.000 & 0.000 & 0.007 & 0.000 & 0.000 & 0.000 & 0.000 & 0.007 & 0.005 & 0.003 & 0.003 & 0.007 & 0.003 & 0.003 & 0.003 & 0.003 & 0.008 & 0.003 \\
\hline 0.01 & MSE & 0.001 & 0.000 & 0.000 & 0.000 & 0.000 & 0.000 & 0.000 & 0.000 & 0.000 & 0.000 & 0.000 & 0.000 & 0.000 & 0.000 & 0.000 & 0.000 & 0.000 & 0.000 & 0.000 & 0.000 \\
\hline 0.05 & BIAS & 0.012 & -0.001 & 0.000 & -0.001 & 0.006 & 0.000 & 0.000 & 0.000 & 0.000 & 0.007 & 0.005 & 0.000 & 0.000 & 0.006 & 0.001 & 0.000 & 0.000 & 0.000 & 0.007 & 0.000 \\
\hline 0.05 & MSE & 0.001 & 0.001 & 0.001 & 0.001 & 0.001 & 0.001 & 0.001 & 0.001 & 0.001 & 0.001 & 0.001 & 0.001 & 0.001 & 0.001 & 0.001 & 0.001 & 0.001 & 0.001 & 0.001 & 0.001 \\
\hline 0.10 & BIAS & 0.011 & -0.001 & 0.000 & -0.001 & 0.005 & 0.000 & 0.000 & 0.000 & 0.000 & 0.006 & 0.004 & -0.001 & -0.001 & 0.005 & 0.000 & 0.000 & 0.000 & 0.000 & 0.006 & 0.000 \\
\hline 0.10 & MSE & 0.002 & 0.002 & 0.002 & 0.002 & 0.002 & 0.002 & 0.002 & 0.002 & 0.002 & 0.002 & 0.002 & 0.002 & 0.002 & 0.002 & 0.002 & 0.002 & 0.002 & 0.002 & 0.002 & 0.002 \\
\hline 0.20 & BIAS & 0.009 & -0.002 & 0.000 & -0.002 & 0.003 & 0.000 & 0.000 & 0.000 & 0.000 & 0.006 & 0.002 & -0.002 & -0.002 & 0.003 & 0.000 & 0.000 & 0.000 & 0.000 & 0.006 & 0.000 \\
\hline 0.20 & MSE & 0.003 & 0.003 & 0.004 & 0.003 & 0.003 & 0.004 & 0.004 & 0.004 & 0.004 & 0.004 & 0.003 & 0.003 & 0.003 & 0.003 & 0.004 & 0.004 & 0.004 & 0.004 & 0.004 & 0.004 \\
\hline 0.30 & BIAS & 0.007 & -0.003 & 0.000 & -0.003 & 0.002 & 0.000 & 0.000 & 0.000 & 0.000 & 0.005 & 0.001 & -0.003 & -0.003 & 0.002 & 0.000 & 0.000 & 0.000 & 0.000 & 0.005 & 0.000 \\
\hline 0.30 & MSE & 0.004 & 0.004 & 0.004 & 0.004 & 0.004 & 0.004 & 0.004 & 0.004 & 0.004 & 0.004 & 0.004 & 0.004 & 0.004 & 0.004 & 0.004 & 0.004 & 0.004 & 0.004 & 0.004 & 0.004 \\
\hline 0.40 & BIAS & 0.005 & -0.003 & 0.000 & -0.003 & 0.001 & 0.000 & 0.000 & 0.000 & 0.000 & 0.004 & -0.001 & -0.003 & -0.003 & 0.001 & 0.000 & 0.000 & 0.000 & 0.000 & 0.004 & 0.000 \\
\hline 0.40 & MSE & 0.004 & 0.004 & 0.004 & 0.004 & 0.004 & 0.004 & 0.004 & 0.004 & 0.004 & 0.004 & 0.004 & 0.004 & 0.004 & 0.004 & 0.004 & 0.004 & 0.004 & 0.004 & 0.004 & 0.004 \\
\hline 0.50 & BIAS & 0.004 & -0.003 & 0.000 & -0.003 & 0.000 & 0.000 & 0.000 & 0.000 & 0.000 & 0.004 & -0.001 & -0.003 & -0.003 & 0.000 & 0.000 & 0.000 & 0.000 & 0.000 & 0.004 & 0.000 \\
\hline 0.50 & MSE & 0.003 & 0.003 & 0.003 & 0.003 & 0.003 & 0.003 & 0.003 & 0.003 & 0.003 & 0.003 & 0.003 & 0.003 & 0.003 & 0.003 & 0.003 & 0.003 & 0.003 & 0.003 & 0.003 & 0.003 \\
\hline 0.60 & BIAS & 0.002 & -0.003 & 0.000 & -0.003 & 0.000 & 0.000 & 0.000 & 0.000 & 0.000 & 0.003 & -0.002 & -0.003 & -0.003 & 0.000 & 0.000 & 0.000 & 0.000 & 0.000 & 0.003 & 0.000 \\
\hline 0.60 & MSE & 0.003 & 0.003 & 0.003 & 0.003 & 0.003 & 0.003 & 0.003 & 0.003 & 0.003 & 0.003 & 0.003 & 0.003 & 0.003 & 0.003 & 0.003 & 0.003 & 0.003 & 0.003 & 0.003 & 0.003 \\
\hline 0.70 & BIAS & 0.001 & -0.003 & 0.000 & -0.003 & -0.001 & 0.000 & 0.000 & 0.000 & 0.000 & 0.002 & -0.002 & -0.003 & -0.003 & -0.001 & 0.000 & 0.000 & 0.000 & 0.000 & 0.002 & 0.000 \\
\hline 0.70 & MSE & 0.002 & 0.002 & 0.002 & 0.002 & 0.002 & 0.002 & 0.002 & 0.002 & 0.002 & 0.002 & 0.002 & 0.002 & 0.002 & 0.002 & 0.002 & 0.002 & 0.002 & 0.002 & 0.002 & 0.002 \\
\hline 0.80 & BIAS & 0.001 & -0.002 & 0.000 & -0.002 & -0.001 & 0.000 & 0.000 & 0.000 & 0.000 & 0.001 & -0.002 & -0.002 & -0.002 & -0.001 & 0.000 & 0.000 & 0.000 & 0.000 & 0.001 & 0.000 \\
\hline 0.80 & MSE & 0.001 & 0.001 & 0.001 & 0.001 & 0.001 & 0.001 & 0.001 & 0.001 & 0.001 & 0.001 & 0.001 & 0.001 & 0.001 & 0.001 & 0.001 & 0.001 & 0.001 & 0.001 & 0.001 & 0.001 \\
\hline 0.90 & BIAS & 0.000 & -0.001 & 0.000 & -0.001 & 0.000 & 0.000 & 0.000 & 0.000 & 0.000 & 0.001 & -0.001 & -0.001 & -0.001 & 0.000 & 0.000 & 0.000 & 0.000 & 0.000 & 0.001 & 0.000 \\
\hline 0.90 & MSE & 0.000 & 0.000 & 0.000 & 0.000 & 0.000 & 0.000 & 0.000 & 0.000 & 0.000 & 0.000 & 0.000 & 0.000 & 0.000 & 0.000 & 0.000 & 0.000 & 0.000 & 0.000 & 0.000 & 0.000 \\
\hline
\end{tabular}


Table S24: Results for sample size $N=150$ and number of predictors $p=5$.

\begin{tabular}{|c|c|c|c|c|c|c|c|c|c|c|c|c|c|c|c|c|c|c|c|c|c|}
\hline$\rho^{2}$ & What & $R^{2}$ & $\mathrm{E}$ & OPE & $\mathrm{S}$ & W & OP1 & $\mathrm{OP} 2$ & OP5 & $\mathrm{P}$ & C & ML & $\mathrm{S}^{+}$ & $\mathrm{E}^{+}$ & $\mathrm{W}^{+}$ & $\mathrm{OP} 1^{+}$ & $\mathrm{OP} 2^{+}$ & $\mathrm{OP}^{+}$ & $\mathrm{P}^{+}$ & $\mathrm{C}^{+}$ & $\mathrm{OPE}^{+}$ \\
\hline 0.00 & BIAS & 0.034 & 0.000 & 0.000 & 0.000 & 0.007 & 0.000 & 0.000 & 0.000 & 0.000 & 0.007 & 0.010 & 0.008 & 0.008 & 0.012 & 0.009 & 0.008 & 0.008 & 0.008 & 0.012 & 0.008 \\
\hline 0.00 & MSE & 0.002 & 0.000 & 0.000 & 0.000 & 0.001 & 0.000 & 0.000 & 0.000 & 0.000 & 0.001 & 0.000 & 0.000 & 0.000 & 0.000 & 0.000 & 0.000 & 0.000 & 0.000 & 0.000 & 0.000 \\
\hline 0.01 & BIAS & 0.033 & 0.000 & 0.000 & 0.000 & 0.007 & 0.000 & 0.000 & 0.000 & 0.000 & 0.007 & 0.007 & 0.005 & 0.005 & 0.010 & 0.006 & 0.006 & 0.006 & 0.006 & 0.010 & 0.006 \\
\hline 0.01 & MSE & 0.002 & 0.001 & 0.001 & 0.001 & 0.001 & 0.001 & 0.001 & 0.001 & 0.001 & 0.001 & 0.001 & 0.001 & 0.000 & 0.001 & 0.001 & 0.001 & 0.001 & 0.001 & 0.001 & 0.001 \\
\hline 0.05 & BIAS & 0.031 & -0.001 & 0.000 & 0.000 & 0.006 & 0.000 & 0.000 & 0.000 & 0.000 & 0.007 & 0.004 & 0.001 & 0.000 & 0.006 & 0.001 & 0.001 & 0.001 & 0.001 & 0.007 & 0.001 \\
\hline 0.05 & MSE & 0.002 & 0.002 & 0.002 & 0.002 & 0.002 & 0.002 & 0.002 & 0.002 & 0.002 & 0.002 & 0.002 & 0.001 & 0.001 & 0.002 & 0.002 & 0.002 & 0.002 & 0.002 & 0.002 & 0.002 \\
\hline 0.10 & BIAS & 0.029 & -0.001 & 0.000 & -0.001 & 0.005 & 0.000 & 0.000 & 0.000 & 0.000 & 0.006 & 0.004 & -0.001 & -0.001 & 0.005 & 0.000 & 0.000 & 0.000 & 0.000 & 0.006 & 0.000 \\
\hline 0.10 & MSE & 0.003 & 0.003 & 0.003 & 0.002 & 0.002 & 0.003 & 0.003 & 0.003 & 0.003 & 0.003 & 0.003 & 0.002 & 0.002 & 0.002 & 0.003 & 0.003 & 0.003 & 0.003 & 0.003 & 0.003 \\
\hline 0.20 & BIAS & 0.025 & -0.002 & 0.000 & -0.002 & 0.004 & 0.000 & 0.000 & 0.000 & 0.000 & 0.006 & 0.002 & -0.002 & -0.002 & 0.004 & 0.000 & 0.000 & 0.000 & 0.000 & 0.006 & 0.000 \\
\hline 0.20 & MSE & 0.004 & 0.004 & 0.004 & 0.004 & 0.004 & 0.004 & 0.004 & 0.004 & 0.004 & 0.004 & 0.004 & 0.004 & 0.004 & 0.004 & 0.004 & 0.004 & 0.004 & 0.004 & 0.004 & 0.004 \\
\hline 0.30 & BIAS & 0.021 & -0.003 & 0.000 & -0.003 & 0.002 & 0.000 & 0.000 & 0.000 & 0.000 & 0.005 & 0.001 & -0.003 & -0.003 & 0.002 & 0.000 & 0.000 & 0.000 & 0.000 & 0.005 & 0.000 \\
\hline 0.30 & MSE & 0.004 & 0.004 & 0.004 & 0.004 & 0.004 & 0.004 & 0.004 & 0.004 & 0.004 & 0.004 & 0.004 & 0.004 & 0.004 & 0.004 & 0.004 & 0.004 & 0.004 & 0.004 & 0.004 & 0.004 \\
\hline 0.40 & BIAS & 0.017 & -0.003 & 0.000 & -0.003 & 0.001 & 0.000 & 0.000 & 0.000 & 0.000 & 0.004 & -0.001 & -0.003 & -0.003 & 0.001 & 0.000 & 0.000 & 0.000 & 0.000 & 0.004 & 0.000 \\
\hline 0.40 & MSE & 0.004 & 0.004 & 0.004 & 0.004 & 0.004 & 0.004 & 0.004 & 0.004 & 0.004 & 0.004 & 0.004 & 0.004 & 0.004 & 0.004 & 0.004 & 0.004 & 0.004 & 0.004 & 0.004 & 0.004 \\
\hline 0.50 & BIAS & 0.014 & -0.003 & 0.000 & -0.003 & 0.000 & 0.000 & 0.000 & 0.000 & 0.000 & 0.003 & -0.002 & -0.003 & -0.003 & 0.000 & 0.000 & 0.000 & 0.000 & 0.000 & 0.003 & 0.000 \\
\hline 0.50 & MSE & 0.003 & 0.004 & 0.004 & 0.004 & 0.003 & 0.004 & 0.004 & 0.004 & 0.004 & 0.003 & 0.003 & 0.004 & 0.004 & 0.003 & 0.004 & 0.004 & 0.004 & 0.004 & 0.003 & 0.004 \\
\hline 0.60 & BIAS & 0.010 & -0.003 & 0.000 & -0.003 & 0.000 & 0.000 & 0.000 & 0.000 & 0.000 & 0.003 & -0.002 & -0.003 & -0.003 & 0.000 & 0.000 & 0.000 & 0.000 & 0.000 & 0.003 & 0.000 \\
\hline 0.60 & MSE & 0.003 & 0.003 & 0.003 & 0.003 & 0.003 & 0.003 & 0.003 & 0.003 & 0.003 & 0.003 & 0.003 & 0.003 & 0.003 & 0.003 & 0.003 & 0.003 & 0.003 & 0.003 & 0.003 & 0.003 \\
\hline 0.70 & BIAS & 0.007 & -0.003 & 0.000 & -0.003 & -0.001 & 0.000 & 0.000 & 0.000 & 0.000 & 0.002 & -0.002 & -0.003 & -0.003 & -0.001 & 0.000 & 0.000 & 0.000 & 0.000 & 0.002 & 0.000 \\
\hline 0.70 & MSE & 0.002 & 0.002 & 0.002 & 0.002 & 0.002 & 0.002 & 0.002 & 0.002 & 0.002 & 0.002 & 0.002 & 0.002 & 0.002 & 0.002 & 0.002 & 0.002 & 0.002 & 0.002 & 0.002 & 0.002 \\
\hline 0.80 & BIAS & 0.005 & -0.002 & 0.000 & -0.002 & -0.001 & 0.000 & 0.000 & 0.000 & 0.000 & 0.001 & -0.002 & -0.002 & -0.002 & -0.001 & 0.000 & 0.000 & 0.000 & 0.000 & 0.001 & 0.000 \\
\hline 0.80 & MSE & 0.001 & 0.001 & 0.001 & 0.001 & 0.001 & 0.001 & 0.001 & 0.001 & 0.001 & 0.001 & 0.001 & 0.001 & 0.001 & 0.001 & 0.001 & 0.001 & 0.001 & 0.001 & 0.001 & 0.001 \\
\hline 0.90 & BIAS & 0.002 & -0.001 & 0.000 & -0.001 & -0.001 & 0.000 & 0.000 & 0.000 & 0.000 & 0.001 & -0.001 & -0.001 & -0.001 & -0.001 & 0.000 & 0.000 & 0.000 & 0.000 & 0.001 & 0.000 \\
\hline 0.90 & MSE & 0.000 & 0.000 & 0.000 & 0.000 & 0.000 & 0.000 & 0.000 & 0.000 & 0.000 & 0.000 & 0.000 & 0.000 & 0.000 & 0.000 & 0.000 & 0.000 & 0.000 & 0.000 & 0.000 & 0.000 \\
\hline
\end{tabular}


Table S25: Results for sample size $N=150$ and number of predictors $p=10$.

\begin{tabular}{|c|c|c|c|c|c|c|c|c|c|c|c|c|c|c|c|c|c|c|c|c|c|}
\hline$\rho^{2}$ & What & $R^{2}$ & $\mathrm{E}$ & $\mathrm{OPE}$ & $\mathrm{S}$ & $\mathrm{W}$ & OP1 & OP2 & OP5 & $\mathrm{P}$ & C & ML & $\mathrm{S}^{+}$ & $\mathrm{E}^{+}$ & $\mathrm{W}^{+}$ & $\mathrm{OP} 1^{+}$ & $\mathrm{OP} 2^{+}$ & $\mathrm{OP}^{+}$ & $\mathrm{P}^{+}$ & $\mathrm{C}^{+}$ & $\mathrm{OPE}^{+}$ \\
\hline 0.00 & BIAS & 0.067 & 0.000 & 0.000 & 0.001 & 0.007 & 0.000 & 0.000 & 0.000 & 0.000 & 0.007 & 0.013 & 0.012 & 0.012 & 0.016 & 0.013 & 0.012 & 0.012 & 0.012 & 0.016 & 0.012 \\
\hline 0.00 & MSE & 0.005 & 0.001 & 0.001 & 0.001 & 0.001 & 0.001 & 0.001 & 0.001 & 0.001 & 0.001 & 0.001 & 0.001 & 0.001 & 0.001 & 0.001 & 0.001 & 0.001 & 0.001 & 0.001 & 0.001 \\
\hline 0.01 & BIAS & 0.066 & 0.000 & 0.000 & 0.000 & 0.007 & 0.000 & 0.000 & 0.000 & 0.000 & 0.007 & 0.010 & 0.009 & 0.009 & 0.013 & 0.009 & 0.009 & 0.009 & 0.009 & 0.013 & 0.009 \\
\hline 0.01 & MSE & 0.005 & 0.001 & 0.001 & 0.001 & 0.001 & 0.001 & 0.001 & 0.001 & 0.001 & 0.001 & 0.001 & 0.001 & 0.001 & 0.001 & 0.001 & 0.001 & 0.001 & 0.001 & 0.001 & 0.001 \\
\hline 0.05 & BIAS & 0.063 & -0.001 & 0.000 & 0.000 & 0.006 & 0.000 & 0.000 & 0.000 & 0.000 & 0.006 & 0.005 & 0.002 & 0.001 & 0.007 & 0.002 & 0.002 & 0.002 & 0.002 & 0.008 & 0.002 \\
\hline 0.05 & MSE & 0.006 & 0.002 & 0.002 & 0.002 & 0.002 & 0.002 & 0.002 & 0.002 & 0.002 & 0.002 & 0.002 & 0.002 & 0.002 & 0.002 & 0.002 & 0.002 & 0.002 & 0.002 & 0.002 & 0.002 \\
\hline 0.10 & BIAS & 0.059 & -0.001 & 0.000 & -0.001 & 0.005 & 0.000 & 0.000 & 0.000 & 0.000 & 0.006 & 0.003 & -0.001 & -0.001 & 0.005 & 0.000 & 0.000 & 0.000 & 0.000 & 0.006 & 0.000 \\
\hline 0.10 & MSE & 0.006 & 0.003 & 0.003 & 0.003 & 0.003 & 0.003 & 0.003 & 0.003 & 0.003 & 0.003 & 0.003 & 0.003 & 0.003 & 0.003 & 0.003 & 0.003 & 0.003 & 0.003 & 0.003 & 0.003 \\
\hline 0.20 & BIAS & 0.052 & -0.002 & 0.000 & -0.002 & 0.004 & 0.000 & 0.000 & 0.000 & 0.000 & 0.005 & 0.002 & -0.002 & -0.002 & 0.004 & 0.000 & 0.000 & 0.000 & 0.000 & 0.005 & 0.000 \\
\hline 0.20 & MSE & 0.006 & 0.004 & 0.004 & 0.004 & 0.004 & 0.004 & 0.004 & 0.004 & 0.004 & 0.004 & 0.004 & 0.004 & 0.004 & 0.004 & 0.004 & 0.004 & 0.004 & 0.004 & 0.004 & 0.004 \\
\hline 0.30 & BIAS & 0.044 & -0.003 & 0.000 & -0.003 & 0.002 & 0.000 & 0.000 & 0.000 & 0.000 & 0.005 & 0.000 & -0.003 & -0.003 & 0.002 & 0.000 & 0.000 & 0.000 & 0.000 & 0.005 & 0.000 \\
\hline 0.30 & MSE & 0.006 & 0.004 & 0.004 & 0.004 & 0.004 & 0.004 & 0.004 & 0.004 & 0.004 & 0.004 & 0.004 & 0.004 & 0.004 & 0.004 & 0.004 & 0.004 & 0.004 & 0.004 & 0.004 & 0.004 \\
\hline 0.40 & BIAS & 0.037 & -0.003 & 0.000 & -0.003 & 0.001 & 0.000 & 0.000 & 0.000 & 0.000 & 0.004 & -0.001 & -0.003 & -0.003 & 0.001 & 0.000 & 0.000 & 0.000 & 0.000 & 0.004 & 0.000 \\
\hline 0.40 & MSE & 0.005 & 0.004 & 0.004 & 0.004 & 0.004 & 0.004 & 0.004 & 0.004 & 0.004 & 0.004 & 0.004 & 0.004 & 0.004 & 0.004 & 0.004 & 0.004 & 0.004 & 0.004 & 0.004 & 0.004 \\
\hline 0.50 & BIAS & 0.030 & -0.003 & 0.000 & -0.003 & 0.000 & 0.000 & 0.000 & 0.000 & 0.000 & 0.003 & -0.002 & -0.003 & -0.003 & 0.000 & 0.000 & 0.000 & 0.000 & 0.000 & 0.003 & 0.000 \\
\hline 0.50 & MSE & 0.004 & 0.004 & 0.004 & 0.004 & 0.004 & 0.004 & 0.004 & 0.004 & 0.004 & 0.004 & 0.004 & 0.004 & 0.004 & 0.004 & 0.004 & 0.004 & 0.004 & 0.004 & 0.004 & 0.004 \\
\hline 0.60 & BIAS & 0.024 & -0.003 & 0.000 & -0.003 & 0.000 & 0.000 & 0.000 & 0.000 & 0.000 & 0.003 & -0.002 & -0.003 & -0.003 & 0.000 & 0.000 & 0.000 & 0.000 & 0.000 & 0.003 & 0.000 \\
\hline 0.60 & MSE & 0.003 & 0.003 & 0.003 & 0.003 & 0.003 & 0.003 & 0.003 & 0.003 & 0.003 & 0.003 & 0.003 & 0.003 & 0.003 & 0.003 & 0.003 & 0.003 & 0.003 & 0.003 & 0.003 & 0.003 \\
\hline 0.70 & BIAS & 0.017 & -0.003 & 0.000 & -0.003 & -0.001 & 0.000 & 0.000 & 0.000 & 0.000 & 0.002 & -0.002 & -0.003 & -0.003 & -0.001 & 0.000 & 0.000 & 0.000 & 0.000 & 0.002 & 0.000 \\
\hline 0.70 & MSE & 0.002 & 0.002 & 0.002 & 0.002 & 0.002 & 0.002 & 0.002 & 0.002 & 0.002 & 0.002 & 0.002 & 0.002 & 0.002 & 0.002 & 0.002 & 0.002 & 0.002 & 0.002 & 0.002 & 0.002 \\
\hline 0.80 & BIAS & 0.011 & -0.002 & 0.000 & -0.002 & -0.001 & 0.000 & 0.000 & 0.000 & 0.000 & 0.001 & -0.002 & -0.002 & -0.002 & -0.001 & 0.000 & 0.000 & 0.000 & 0.000 & 0.001 & 0.000 \\
\hline 0.80 & MSE & 0.001 & 0.001 & 0.001 & 0.001 & 0.001 & 0.001 & 0.001 & 0.001 & 0.001 & 0.001 & 0.001 & 0.001 & 0.001 & 0.001 & 0.001 & 0.001 & 0.001 & 0.001 & 0.001 & 0.001 \\
\hline 0.90 & BIAS & & & & -0.001 & -0.001 & & & & 0.000 & & -0.001 & & & -0.001 & & 0.000 & 0.000 & & 0.001 & 0.000 \\
\hline 0.90 & MSE & 0.000 & 0.000 & 0.000 & 0.000 & 0.000 & 0.000 & 0.000 & 0.000 & 0.000 & 0.000 & 0.000 & 0.000 & 0.000 & 0.000 & 0.000 & 0.000 & 0.000 & 0.000 & 0.000 & 0.000 \\
\hline
\end{tabular}

